\title{
SATURATION PROPERTIES OF IDEALS IN GENERIC EXTENSIONS. II
}

\author{
BY
}

JAMES E. BAUMGARTNER ${ }^{1}$ AND ALAN D. TAYLOR ${ }^{2}$

\begin{abstract}
The general type of problem considered here is the following. Suppose $I$ is a countably complete ideal on $\omega_{1}$ satisfying some fairly strong saturation requirement (e.g. $I$ is precipitous or $\omega_{2}$-saturated), and suppose that $P$ is a partial ordering satisfying some kind of chain condition requirement (e.g. $P$ has the c.c.c. or forcing with $P$ preserves $\omega_{1}$ ). Does it follow that forcing with $P$ preserves the saturation property of $I$ ? In this context we consider not only precipitous and $\omega_{2}$-saturated ideals, but we also introduce and study a class of ideals that are characterized by a property lying strictly between these two notions. Some generalized versions of Chang's conjecture and Kurepa's hypothesis also arise naturally from these considerations.
\end{abstract}

0. Introduction. This paper continues the study begun in [BT] of saturation properties of ideals in generic extensions. The distinction between the present work and its precursor is that the saturation properties of ideals that we now consider are all strong in the sense of implying the consistency of some large cardinals.

Our notation and terminology is explained in $\S 1$, while the main results of the paper begin in $\$ 2$ with a consideration of the following question. Is the $\omega_{2}$-saturation of a countably complete ideal on $\omega_{1}$ preserved under countable chain condition forcing? Although this question is still open, we formulate some rather useful equivalents involving Boolean ultrapowers, a generalized version of Chang's conjecture, and a generalized version of Kurepa's hypothesis.

Using these equivalents, we show in $\$ 3$ that one obtains an affirmative answer to the above question if "c.c.c." is replaced by " $\sigma$-finite c.c.". Unfortunately, it turns out that the variant of the Galvin-Hajnal ordering which we used in a similar situation in [BT] will not suffice to settle the present question. $\$ 3$ also contains the following "nonpreservation" result: if one forces with the partial ordering for adding a closed unbounded subset of $\omega_{1}$ with finite conditions, then in the extension there are no $\omega_{2}$-saturated countably complete ideals on $\omega_{1}$.

In $\$ 4$ we study the class of countably complete ideals on $\omega_{1}$ that are (simultaneously) precipitous and " $\omega_{2}$-preserving" (i.e. forcing with $\mathscr{P}\left(\omega_{1}\right) / I$ does not collapse $\left.\omega_{2}\right)$. We refer to these ideals as "presaturated", and some of their basic properties are established here. For example, we show that every presaturated ideal

Received by the editors May 21, 1980 and, in revised form, May 26, 1981.

1980 Mathematics Subject Classification. Primary 02K05; Secondary 04A20.

'Research partially supported by NSF grant MCS 79-03376.

${ }^{2}$ Research partially supported by NSF grant MCS 77-04147. 
on $\omega_{1}$ is a weak $P$-point and (hence) if there is a presaturated ideal on $\omega_{1}$, then there is a normal presaturated ideal on $\omega_{1}$.

$\S 5$ is primarily concerned with the preservation of presaturation. For example, it is shown here that the existence of a presaturated countably complete ideal on $\omega_{1}$ is preserved when forcing with the ordering for adding a closed unbounded subset of $\omega_{1}$ with finite conditions. This result, when combined with the "nonpreservation" theorem in $\S 3$, shows that the existence of a presaturated ideal on $\omega_{1}$ does not entail the existence of an $\omega_{2}$-saturated countably complete ideal on $\omega_{1}$. (It also turns out that the existence of a precipitous ideal on $\omega_{1}$ does not imply the existence of a presaturated ideal on $\omega_{1}$.) The main theorem in $\$ 5$, however, is sufficiently general to yield several additional results of interest. For example, we also obtain here a strengthening of Kakuda's recent result that the precipitousness of a $\kappa$-complete ideal on $\kappa$ is preserved under $\kappa$-c.c. forcing.

$\$ 6$ contains several questions suggested by the considerations in this paper.

We would like to thank the referee for several suggestions that have been incorporated into the final version of this paper.

1. Notation and terminology. Our set-theoretic notation and terminology is standard. If $X$ is a set, then $\mathscr{P}(X)$ is the power set of $X$ and $|X|$ is the cardinality of $X$. If $X$ is a set and $\kappa$ is a cardinal, then $[X]^{\kappa}=\{Y \subseteq X:|Y|=\kappa\}$ and $[X]^{<\kappa}=\{Y \subseteq X$ : $|Y|<\kappa\}$.

A partially ordered set $P$ satisfies the $\mu$-chain condition ( $\mu$-c.c.) iff every pairwise incompatible subset of $P$ is of cardinality less than $\mu$. The $\omega_{1}$-chain condition is called the countable chain condition (c.c.c.). $P$ has the $\mu$-finite chain condition iff there is a function $f: P \rightarrow \mu$ such that for each $\alpha<\mu$, every pairwise incompatible subset of $f^{-1}(\{\alpha\})$ is finite. The $\omega$-finite chain condition is usually called the $\sigma$-finite chain condition ( $\sigma$-finite c.c.).

An ideal $I$ on $\kappa$ is said to be $\lambda$-complete if it is closed under unions of size less than $\lambda$. If $I$ is $\omega_{1}$-complete, then $I$ is also called countably complete. Unless explicitly stated otherwise, we will use the phrase "ideal on $\kappa$ " to mean one that is countably complete, proper (i.e. $\kappa \notin I$ ), and contains all singleton subsets of $\kappa$. If $I$ is an ideal on $\kappa$, then $I^{+}$denotes the collection of sets of positive $I$-measure; i.e. $I^{+}=\stackrel{\rho}{ }(\kappa)-I$, and $I^{*}$ denotes the collection of sets of $I$-measure one; i.e. $I^{*}=\{X \subseteq \kappa: \kappa-X \in I\}$. If $A \in I^{+}$, then the restriction of $I$ to $A$ is the ideal $I \mid A=\{X \subseteq \kappa: X \cap A \in I\}$. An ideal $I$ on $\kappa$ is said to be normal if every regressive function defined on a set of positive $I$-measure is constant on a set of positive $I$-measure. If $\kappa$ is a regular uncountable cardinal, then $I_{\kappa}$ denotes the ideal consisting of all subsets of $\kappa$ of cardinality less than $\kappa$, and $N S_{\kappa}$ denotes the (normal) ideal of all nonstationary subsets of $\kappa$. Notice that $\left(N S_{\kappa}\right)^{*}$ is the filter generated by the set of closed unbounded subsets of $\kappa$.

If $I$ is an ideal on $\kappa$, then two sets $A$ and $B$ in $I^{+}$are said to be $I$-almost disjoint iff $A \cap B \in I$. The ideal $I$ is $\lambda$-saturated iff every pairwise $I$-almost disjoint collection $F \subseteq I^{+}$is of cardinality less than $\lambda$. Note that $I$ is $\lambda$-saturated iff the Boolean algebra $\mathcal{P}(\kappa) / I$ satisfies the $\lambda$-chain condition. For more background on the theory of ideals, see [BTW, JP, $\left.\mathbf{T}_{1}\right]$ or $\left[\mathbf{T}_{\mathbf{2}}\right]$. 
Our forcing notation is reasonably standard, but notice that we write $p \leqslant q$ to mean that $p$ contains more information than $q$. We also freely pass from a partially ordered set to its completion, thus taking arbitrary infs and sups in forcing arguments whenever it is convenient. We generally discuss forcing as if it is taking place over the universe of set theory $V$. The reader uncomfortable with this approach can easily recast these results in terms of a sufficiently large fragment of ZFC. If $x \in V$, then we will use $x$ itself as a term of the language of forcing to denote $x$. Other terms, i.e. terms not denoting specific elements of $V$, will be denoted by letters with a dot over the top. If $\dot{x}$ is a term, then the object denoted or represented by $\dot{x}$ in the generic extension $V[G]$ is written $\dot{x}^{V[G]}$.

Of course, several rather elementary facts from the theory of forcing will be used throughout this paper with very little comment. One such fact (which is crucial for our present considerations) is the observation that if $I$ is a countably complete ideal on $\kappa$, then $I$ generates a countably complete (proper) ideal on $\kappa$ in any c.c.c. generic extension. This observation is elaborated on in the beginning of $\S 5$.

On several occasions, we will refer to the ordering $P$ for "adding a closed unbounded subset of $\omega_{1}$ with finite conditions". This is an ordering invented by the first named author to settle some questions in general topology $\left[\mathbf{B}_{1}\right]$. There are several (equivalent) ways to describe $P$; perhaps the simplest is to let $P$ consist of all finite functions $p$ mapping $\omega_{1}$ into $\omega_{1}$ for which there is a countable closed set $C \subseteq \omega_{1}$ so that if $h$ enumerates $C$ in increasing order, then $p \subseteq h$. (Of course, for $p_{1}, p_{2} \in P$ we set $p_{1} \leqslant p_{2}$ iff $p_{1} \supseteq p_{2}$.) It is, however, sometimes useful to have the following alternate characterization of $P$. If $\alpha$ is an ordinal let $\alpha=\omega^{\alpha_{1}}+\omega^{\alpha_{2}}$ $+\cdots+\omega^{\alpha_{n}}$ be the unique decomposition of $\alpha$ as a sum of indecomposable ordinals (powers of $\omega$ ) with $\alpha_{1} \geqslant \cdots \geqslant \alpha_{n}$. If $\beta=\omega^{\beta_{1}}+\cdots+\omega^{\beta_{m}}$, then we say $\alpha$ dominates $\beta$ iff $\alpha_{n} \geqslant \beta_{m}$. Now $P$ may be defined as the set of all finite increasing functions from $\omega_{1}$ to $\omega_{1}$ such that, for all $\beta, p(\beta)$ dominates $\beta$. Since ordinal exponentiation is absolute, so is the dominance relation; hence so is the definition of $P$. This shows also that if $p \in P$, range $(p) \subseteq \gamma$ and $\gamma$ is indecomposable, then $p \cup\{(\gamma, \gamma)\} \in P$.

We will include here (for the sake of completeness) a proof that forcing with $P$ does not collapse $\omega_{1}$. It should be remarked that the following proof easily extends to show that $P$ is proper (i.e. that forcing with $P$ does not destroy any stationary subset of $[\kappa]^{\omega}$ for $\left.\kappa>\omega\right)$; for this extension one uses the fact that for every closed unbounded set $C \subseteq[\kappa]^{\omega}$ there is a function $f:[\kappa]^{<\omega} \rightarrow \kappa$ so that $\left\{X \in[\kappa]^{\omega}: X\right.$ is closed under $f\} \subseteq C$. (See $\S 3$ of $[$ BTT].)

So suppose that $p \in P$ and $p \Vdash \dot{f}: \omega \rightarrow \omega_{1}$. We inductively construct an increasing sequence $\left\langle\gamma_{n}: n \in \omega\right\rangle$ of indecomposable ordinals as follows. Let $\gamma_{0}$ be any countable indecomposable ordinal so that $\gamma_{0} \geqslant \sup (\operatorname{range}(p))$. Given $\gamma_{n}$, choose $\gamma_{n+1}$ to be an indecomposable ordinal greater than $\gamma_{n}$ and large enough so that for any $q \leqslant p$ with range $(q) \subseteq \gamma_{n}$, and for any $k \in \omega$ there is some $q^{\prime} \leqslant q$ with $\operatorname{range}\left(q^{\prime}\right) \subseteq \gamma_{n+1}$ and such that $q^{\prime} \mid r \dot{f}(k) \leqslant \gamma_{n+1}$. Now let $\gamma=\sup \left\{\gamma_{n}: n \in \omega\right\}$. Then $\gamma$ is indecomposable and so $p \cup\{(\gamma, \gamma)\} \in P$. But now it is a straightforward matter to verify that $p \cup\{(\gamma, \gamma)\} \mid \vdash$ " $\forall k \in \omega(\dot{f}(k)<\gamma)$ ".

Notice that if $G$ is a $P$-generic set for the ordering $P$ described above, then $\cup G$ is the enumerating function for a closed unbounded set $C \subseteq \omega_{1}$ in the extension $V[G]$. 
One of the crucial properties of this type of forcing is that this new closed unbounded set $C$ contains no countable set in $V$ as a subset.

We will make considerable use of the so-called "generic ultrapower" (or "Boolean ultrapower") construction originally introduced by Solovay [S]. We will fix our notation regarding this here and mention a couple of rather crucial basic facts; the reader desiring a more thorough background should consult $[\mathbf{J}, \mathbf{J P}]$, or $[\mathbf{S}]$.

If $I$ is an ideal on $\omega_{1}$, then we can force with the Boolean algebra $B(I)=\mathscr{P}\left(\omega_{1}\right) / I$ to obtain a generic ultrafilter $U$. If $X \in I^{+}$then $[X]$ will denote the corresponding equivalence class in $B(I)$. Now, in $V[U]$, we can form the ultrapower $V^{\omega_{1}} / U$ made up of equivalence classes $[f]$ where $f: \omega_{1} \rightarrow V$ and $f \in V$. The ideal $I$ is said to be precipitous iff this ultrapower is always well founded, and in this case we identify $V^{\omega_{1}} / U$ with its transitive collapse. The letter $j$ will denote the canonical elementary embedding of $V$ into $V^{\omega_{1}} / U$; i.e. $j(x)=\left[f_{x}\right]$ where $f_{x}(\alpha)=x$ for every $\alpha<\omega_{1}$. Finally, in extended discussions (e.g. §5) where we have under consideration a fixed ideal $I$ and a fixed $B(I)$-generic ultrafilter $U$, we will (for typographical convenience) use " $M$ " in place of " $V{ }^{\omega_{1}} / U$ ".

If the ideal $I$ is $\omega_{2}$-saturated, then there are several nice properties arising in the above construction. For example, $B(I)$ is a complete Boolean algebra and $V^{\omega_{1}} / U$ is well founded (i.e. $I$ is precipitous). Moreover, $V[U]$ and (the collapse of) $V^{\omega_{1}} / U$ have the same countable sets of ordinals, and if $X \in I^{+}$and $[X] \vdash \dot{y} \in V^{\omega_{1}} / U$, then there is some $f: \omega_{1} \rightarrow V$ so that $[X] \vdash \dot{y}=[f]$. Proofs of all these assertions can be found in $[\mathbf{J}]$.

2. Equivalents for preservation of $\omega_{2}$-saturation. In this section we investigate the preservation of $\omega_{2}$-saturated ideals on $\omega_{1}$ under partial orderings $P$ with the countable chain condition. That is, for such $I$ and $P$, we ask when it is true that

$\vdash_{P}$ "The ideal generated by $I$ is $\omega_{2}$-saturated".

There are two main results. The first considers both the Boolean ultrapower construction and a generalized version of Kurepa's hypothesis in order to give necessary and sufficient conditions for a given c.c.c. partial ordering to preserve the $\omega_{2}$-saturation of a given ideal on $\omega_{1}$. The second uses a generalized version of Chang's conjecture to give necessary and sufficient conditions for the $\omega_{2}$-saturation of a given ideal $I$ on $\omega_{1}$ to be preserved by every c.c.c partial ordering.

If $I$ is an ideal on $\omega_{1}$, then the transversals hypothesis for $I$, denoted $T H(I)$, is the assertion that there is a family $\left\{g_{\alpha}: \alpha<\omega_{2}\right\}$ of functions mapping $\omega_{1}$ to $\omega$ such that if $\alpha<\beta$ then $\left\{\xi \in \omega_{1}: g_{\alpha}(\xi)=g_{\beta}(\xi)\right\} \in I$. The transversals hypothesis for $\omega_{1}$ is just $T H\left(I_{\omega_{1}}\right)$; it follows easily from Kurepa's hypothesis, but it is known to be strictly weaker. It is well known and easy to see that if $I$ is $\omega_{2}$-saturated then $T H(I \mid A)$ fails for every $A \in I^{+}$. For more on $T H(I)$, see Chapter 3 , $\S 4$ of [BTW].

The following further weakening of Kurepa's hypothesis will yield a purely combinatorial equivalent of the preservation of the $\omega_{2}$-saturation of an ideal by a c.c.c. partial ordering. 
Definition 2.1. If $I$ is an ideal on $\omega_{1}$ and $P$ is a partial ordering, then $T H(I, P)$ denotes the assertion that there exists a family $\left\{g_{\alpha}: \alpha<\omega_{2}\right\}$ of functions mapping $\omega_{1}$ to $P$ such that if $\alpha<\beta<\omega_{2}$ then $\left\{\xi<\omega_{1}: g_{\alpha}(\xi)\right.$ is compatible with $\left.g_{\beta}(\xi)\right\} \in I$.

Notice that if $P$ is a countable antichain, then $T H(I, P)$ reduces to just $T H(I)$. We will make use of the trivial observation that for $T H(I, P)$ to hold it suffices to have each $g_{\alpha}$ defined only on a set of $I$-measure one.

THEOREM 2.2. Suppose $I$ is an $\omega_{2}$-saturated ideal on $\omega_{1}$ and $P$ has the countable chain condition. Then the following are equivalent:

(a) $\vdash_{P}$ "I generates an $\omega_{2}$-saturated ideal".

(b) $T H(I \mid A, P)$ fails for every $A \in I^{+}$.

(c) $\Vdash_{B(I)}$ " $j(P)$ has the countable chain condition".

Proof. (a) implies (b): Suppose that $A \in I^{+}$and $\left\{g_{\alpha}: \alpha<\omega_{2}\right\}$ shows that $T H(I \mid A, P)$ holds. Let $\dot{G}$ denote the canonical $P$-generic set over $V$. For $\alpha<\omega_{2}$, let $\dot{A}_{\alpha}=\left\{\xi<\omega_{1}: g_{\alpha}(\xi) \in \dot{G}\right\}$, and let $\dot{I}$ denote the ideal generated by $I$. Then clearly If $_{P}$ “ $\left\langle\dot{A}_{\alpha}: \alpha<\omega_{2}\right\rangle$ is an $\dot{I}$-almost disjoint family".

We assert that for each $\alpha<\omega_{2}$ there is $p_{\alpha} \in P$ such that $p_{\alpha} \Vdash \dot{A}_{\alpha} \in \dot{I}^{+}$. Let $\left\langle\left(q_{n}, B_{n}\right): n \in \omega\right\rangle$ be a maximal family such that $B_{n} \in I, q_{n} \Vdash \dot{A}_{\alpha} \subseteq B_{n}$, and if $m \neq n$ then $q_{m}$ and $q_{n}$ are incompatible. Choose $\xi \in A-\cup\left\{B_{n}: n \in \omega\right\}$ and let $p_{\alpha}=g_{\alpha}(\xi)$. Then $p_{\alpha}$ works.

Since $P$ has the c.c.c. we know $\wedge_{\beta<\omega_{2}}\left(\vee_{\beta<\alpha} p_{\alpha}\right) \neq 0$. Choose $p \leqslant$ $\wedge_{\beta<\omega_{2}}\left(\vee_{\beta<\alpha} p_{\alpha}\right)$. Then it is easy to see that for every $q \leqslant p$ and $\beta<\omega_{2}$ there is some $\alpha<\omega_{2}$ such that $\beta<\alpha$ and $q$ is compatible with $p_{\alpha}$. But then

$$
p|+| \dot{I}^{+} \cap\left\{\dot{A}_{\alpha}: \alpha<\omega_{2}\right\} \mid=\omega_{2},
$$

contradicting (a).

(b) implies (c): If $A \in I^{+}$, let [ $\left.A\right]$ denote its equivalence class in $B(I)$. Let $\lambda=\omega_{2}$. Then if $U$ is $B(I)$-generic over $V, \lambda$ is the $\omega_{1}$ of $V[U]$. Now suppose that (c) fails and $[A] \mid \vdash "\left\langle\dot{p}_{\alpha}: \alpha<\lambda\right\rangle$ is a sequence of pairwise incompatible elements of $j(P)$ ". For each $\alpha<\lambda$ there is $f_{\alpha}: \omega_{1} \rightarrow P$ such that $\left\langle f_{\alpha}: \alpha<\lambda\right\rangle \in V$ and $[A] \Vdash \forall \alpha\left(\dot{p}_{\alpha}=\left[f_{\alpha}\right]\right)$. But then since $A \in(I \mid A)^{*}$, it is easy to see that $\left\langle f_{\alpha}: \alpha<\omega_{2}\right\rangle$ shows that $\operatorname{TH}(I \mid A, P)$ holds, and hence (b) fails.

(c) implies (a): Suppose that (a) fails. Then for some $p \in P$ we have $p \Vdash " ~\left\langle\dot{A}_{\alpha}\right.$ : $\left.\alpha<\omega_{2}\right\rangle$ is a family of $\dot{I}$-almost disjoint elements of $\dot{I}^{+}$". For each $\alpha<\omega_{2}$, let $B_{\alpha}=\left\{\xi \in \omega_{1}: \exists q \leqslant p\left(q \Vdash \xi \in \dot{A}_{\alpha}\right)\right\}$. Since $p \Vdash \dot{A} \in \dot{I}^{+}$, we must have $B_{\alpha} \in I^{+}$. Since $B(I)$ has the $\omega_{2}$-chain condition, it follows that we can choose $[A] \leqslant$ $\wedge_{\beta<\omega_{2}}\left(\bigvee_{\beta<\alpha}\left[B_{\alpha}\right]\right)$ so that $[A] \neq 0$. Then

(*) $\quad(\forall[B] \leqslant[A])\left(\forall \beta<\omega_{2}\right)\left(\exists \alpha<\omega_{2}\right)\left(\beta<\alpha\right.$ and $\left.[B] \wedge\left[B_{\alpha}\right] \neq 0\right)$.

But now if $f_{\alpha}: \omega_{1} \rightarrow P$ is defined so that for every $\xi \in B_{\alpha}, f_{\alpha}(\xi) \leqslant p$ and $f_{\alpha}(\xi) \Vdash \xi \in \dot{A}_{\alpha}$, then since $p$ เ $\dot{A}_{\alpha} \cap \dot{A}_{\beta} \in \dot{I}$ for $\alpha \neq \beta$, we have $\left.\left[B_{\alpha}\right] \wedge\left[B_{\beta}\right] \Vdash \Vdash \mid f f_{\alpha}\right]$ and $\left[f_{\beta}\right]$ are incompatible", assuming $B_{\alpha} \cap B_{\beta} \in I^{+}$. On the other hand, if $\dot{U}$ denotes the canonical $B(I)$-generic set, then by $(*),[A] \mid{ }^{\prime}$ “ $\dot{X}=\left\{\alpha:\left[B_{\alpha}\right] \in \dot{U}\right\}$ is uncountable". But then $[A] \mid \vdash "\left\langle\left[f_{\alpha}\right]: \alpha \in \dot{X}\right\rangle$ is an uncountable antichain in $j(P)$ ", as desired. 
REMARKS. 1. A still further (apparent) weakening of these Kurepa-type properties is also possible. That is, let $w T H(I)$, " $w$ " for "weak", denote the modified assertion obtained by replacing " $g_{\alpha}: \omega_{1} \rightarrow \omega$ " by " $g_{\alpha}: A_{\alpha} \rightarrow \omega$ and $A_{\alpha} \in I^{+}$", and let $w T H(I, P)$ denote the corresponding modification of $T H(I, P)$. The interest in these notions is that we then have the following:

(a) $w T H(I)$ fails iff $I$ is $\omega_{2}$-saturated.

(b) $w T H(I, P)$ fails iff $P$ is c.c.c. and $\vdash_{P}$ " $I$ is $\omega_{2}$-saturated".

The proof of (b) involves showing that if $w T H(I \mid A, P)$ holds and $I$ is $\omega_{2}$-saturated, then $T H(I \mid A, P)$ holds for some $A \in I^{+}$. We leave the details for the reader.

2. The equivalence of (a) and (c) can be restated in a way that emphasizes the symmetry involved. That is, if $I$ is an ideal on $\omega_{1}$ and $P$ is a partial ordering, then the following are equivalent:

(i) $B(I)$ has the $\omega_{2}$-c.c. and $B(I) \vdash^{\text {" }} j(P)$ has the $\omega_{1}$-c.c.".

(ii) $P$ has the $\omega_{1}$-c.c. and $P \mid r$ " $B(I)$ has the $\omega_{2}$-c.c.".

Notice that in this restatement, we have moved the chain condition requirements on $B(I)$ and $P$ from the hypothesis of the theorem to a part of the equivalences in the conclusion. The required addition to the proof is trivial and omitted.

We now turn our attention to the question of what conditions on an ideal $I$ on $\omega_{1}$ are sufficient (and necessary) to insure that the $\omega_{2}$-saturation of $I$ is preserved when forcing with any c.c.c. ordering $P$. Our starting point is a discussion of Chang's conjecture and its generalization to the context of ideals.

Chang's conjecture may be stated in combinatorial terms as $\omega_{2} \rightarrow\left[\omega_{1}\right]_{\omega_{1 / \omega}}^{<\omega}$, i.e. if $f$ : $\left[\omega_{2}\right]^{<\omega} \rightarrow \omega_{1}$ then there is $X \in\left[\omega_{2}\right]^{\omega_{1}}$ such that $\left|f\left([X]^{<\omega}\right)\right| \leqslant \omega$. It seems to be part of the folklore that this is equivalent to the weaker-looking proposition $\omega_{2} \rightarrow\left[\omega_{1}\right]_{\omega_{1 / \omega}}^{2}$. The proof runs as follows: First, for each $\alpha \in \omega_{2}$ fix a well-ordering $<_{\alpha}$ of $\alpha$ in order-type $\leqslant \omega_{1}$. Now, given $f:\left[\omega_{2}\right]^{<\omega} \rightarrow \omega_{1}$, define $g:\left[\omega_{2}\right]^{2} \rightarrow \omega_{1}$ as follows. If $\alpha<\beta$ and the set of $<_{\beta}$-predecessors of $\alpha$ is the (countable) set $X_{\alpha \beta}$, then let $g(\{\alpha, \beta\})=\sup \left\{f(x): x \in\left[X_{\alpha \beta} \cup\{\alpha, \beta\}\right]^{<\omega}\right\}$. Suppose $X \in\left[\omega_{2}\right]^{\omega_{1}}, \xi<\omega_{1}$, and $g\left([X]^{2}\right) \subseteq \xi$. Let $x=\left\{\alpha_{1}, \ldots, \alpha_{n}\right\} \in[X]^{<\omega}$. Assume that $\max (x)=\alpha_{n}$ and the $<_{\alpha_{n}}$-largest element of $x-\left\{\alpha_{n}\right\}$ is $\alpha_{i}$. Then clearly $f(x) \leqslant g\left(\left\{\alpha_{i}, \alpha_{n}\right\}\right)$, so $f\left([X]^{<\omega}\right)$ $\subseteq \xi$

Thus the next definition is a generalization of Chang's conjecture to the context of ideals.

Definition 2.3. Suppose $I$ is an ideal on $\omega_{1}$.

(a) $\omega_{2} \rightarrow\left[\omega_{1}\right]_{I^{*}}^{2}$ means: if $f:\left[\omega_{2}\right]^{2} \rightarrow I^{*}$ then there exists $X \in\left[\omega_{2}\right]^{\omega_{1}}$ such that $\cap f\left([X]^{2}\right) \neq 0$.

(b) We say that $I$ satisfies Chang's conjecture iff $\omega_{2} \rightarrow\left[\omega_{1}\right]_{(I \mid A)^{*}}^{2}$ for every $A \in I^{+}$.

It is easy to see that Chang's conjecture holds iff $I_{\omega_{1}}$ satisfies Chang's conjecture. This is the motivation for Definition 2.3, except that this would also have been achieved if we had chosen to replace " $\neq 0$ " in $2.3(\mathrm{a})$ with " $\in I^{*}$ ". The following observation of Laver dictated our choice: there is a function $f:\left[\omega_{2}\right]^{2} \rightarrow N S_{\omega_{1}}^{*}$ such that for every $X \in\left[\omega_{2}\right]^{\omega_{1}}$ we have $\left|\cap f\left([X]^{2}\right)\right| \leqslant 1$. (To see this, let $\left\langle f_{\alpha} \cdot \alpha<\omega_{2}\right\rangle$ be functions from $\omega_{1}$ to $\omega_{1}$ so that if $\alpha \neq \beta$ then $A_{\alpha \beta}=\left\{\xi: f_{\alpha}(\xi)=f_{\beta}(\xi)\right\} \in I$. For $\alpha<\omega_{2}$ let $C_{\alpha}$ be closed unbounded so that each $\xi \in C_{\alpha}$ is closed under $f_{\alpha}$. Now set 
$f(\{\alpha, \beta\})=\left(C_{\alpha} \cap C_{\beta}\right)-A_{\alpha \beta}$. $)$ Another observation (whose proof is left for the reader) is that if $I$ is $\omega_{2}$-saturated, then $\omega_{2} \rightarrow[\alpha]_{I^{*}}^{2}$ for every $\alpha<\omega_{1}$. The notation here should be obvious. The question of whether this result, or Laver's result above, can be strengthened in the obvious way leads us to the second main theorem of this section.

THEOREM 2.4. For an $\omega_{2}$-saturated ideal I on $\omega_{1}$, the following are equivalent.

(a) I satisfies Chang's conjecture.

(b) For every c.c.c. partial ordering $P, \vdash_{P}$ "I generates an $\omega_{2}$-saturated ideal".

Proof. (a) implies (b): Suppose that (b) fails and that $P$ is a c.c.c. ordering that does not force the ideal generated by $I$ to be $\omega_{2}$-saturated. Then by Theorem 2.2, we know that $T H(I \mid A, P)$ holds for some $A \in I^{+}$. Let $\left\langle g_{\alpha}: \alpha<\omega_{2}\right\rangle$ witness this. Now define $f:\left[\omega_{2}\right]^{2} \rightarrow(I \mid A)^{*}$ by $f(\{\alpha, \beta\})=\left\{\xi \in \omega_{1}: g_{\alpha}(\xi)\right.$ is compatible with $\left.g_{\beta}(\xi)\right\}$. Then $f$ shows that $\omega_{2} \nrightarrow\left[\omega_{1}\right]_{(I \mid A)^{*}}^{2}$ (and hence $I$ does not satisfy Chang's conjecture), since if $X \subseteq \omega_{2}$ and $\xi \in \cap f\left([X]^{2}\right)$ then $\left\{g_{\alpha}(\xi): \alpha \in X\right\}$ is an antichain in $P$ and hence it (and $X$ ) must thus be countable.

(b) implies (a): Suppose $I$ does not satisfy Chang's conjecture. Fix $A \in I^{+}$and $f$ : $\left[\omega_{2}\right]^{2} \rightarrow(I \mid A)^{*}$ such that $\cap f\left([X]^{2}\right)=0$ whenever $X \in\left[\omega_{2}\right]^{\omega_{1}}$. For each $\beta<\omega_{2}$, let $k_{\beta}: \beta \rightarrow \omega_{1}$ be one-to-one. If $\alpha<\beta<\omega_{2}$ then let

$$
g(\{\alpha, \beta\})=\bigcap\left\{f(\{\alpha, \gamma\}): k_{\beta}(\gamma)<k_{\beta}(\alpha)\right\} \cap f(\{\alpha, \beta\}) .
$$

Note that $g(\{\alpha, \beta\}) \in(I \mid A)^{*}$ and that if $\alpha<\beta<\gamma$ then either $g(\{\alpha, \gamma\}) \subseteq f(\{\alpha, \beta\})$ or $g(\{\beta, \gamma\}) \subseteq f(\{\alpha, \beta\})$.

Now we will generically add a sequence $\left\langle A_{\alpha}: \alpha<\omega_{2}\right\rangle$ that will witness the failure of the $\omega_{2}$-saturation of the ideal generated by $I$ in the extension. A condition will be a finite piece of information about which $\xi$ 's from $\omega_{1}$ will be in which $A_{\alpha}$ 's. Trivial density arguments will insure that each $A_{\alpha}$ is of positive measure, but to guarantee that $A_{\alpha} \cap A_{\beta} \in \dot{I}$ for $\alpha \neq \beta$, we will add the stipulation that if some condition forces $\xi \in \dot{A}_{\alpha}$ and $\xi \in \dot{A}_{\beta}$ for $\alpha \neq \beta$, then $\xi \notin g(\{\alpha, \beta\})$. Of course the crux of the matter is to verify that this notion of forcing has the c.c.c.; this is why the "counterexample" $f$ was replaced by the stronger "counterexample" $g$.

More formally, let $P=\left\{x \in\left[A \times \omega_{2}\right]^{<\omega}\right.$ : if $(\xi, \alpha),(\xi, \beta) \in x$ and $\alpha \neq \beta$, then $\xi \notin g(\{\alpha, \beta\})\}$. Let $x \leqslant y$ iff $y \subseteq x$. Suppose we know that $P$ has the c.c.c. If $G$ is $P$-generic over $V$, then in $V[G]$ define $\left\langle A_{\alpha}: \alpha<\omega_{2}\right\rangle$ by setting $A_{\alpha}=\left\{\xi<\omega_{1}\right.$ : $(\xi, \alpha) \in \cup G\}$. It is clear by genericity that if $J$ is the ideal generated by $I$ in $V[G]$, then $\left\langle A_{\alpha}: \alpha<\omega_{2}\right\rangle$ is a $J$-almost disjoint family of elements of $J^{+}$.

Thus, we need only show that $P$ has the c.c.c. Suppose $Z=\left\{z_{\beta}: \beta<\omega_{1}\right\}$ is an uncountable antichain. Without loss of generality we can assume that the elements of $Z$ all have cardinality $n$. Say $z_{\beta}=\left\{\left(\xi_{1}^{\beta}, \alpha_{1}^{\beta}\right), \ldots,\left(\xi_{n}^{\beta}, \alpha_{n}^{\beta}\right)\right\}$. Furthermore, we may assume that if $1 \leqslant i \leqslant n$ then either all the $\alpha_{i}^{\beta}$ are equal or else $\beta<\gamma$ implies $\alpha_{i}^{\beta}<\alpha_{i}^{\gamma}$; similarly for the $\xi_{i}^{\beta}$. Note that since $z_{\beta}$ and $z_{\gamma}$ are incompatible there must be $i, j$ such that $\xi_{i}^{\beta}=\xi_{j}^{\gamma}$ and $\xi_{i}^{\beta} \in g\left(\left\{\alpha_{i}^{\beta}, \alpha_{j}^{\gamma}\right\}\right)$. We say $z_{\beta}, z_{\gamma}$ disagree at $(i, j)$.

Let $D$ be a uniform ultrafilter on $\omega_{1}$ and for $1 \leqslant i, j \leqslant n$, let $B_{i j}=\left\{\beta:\left\{\gamma: z_{\beta}\right.\right.$ and $z_{\gamma}$ disagree at $\left.\left.(i, j)\right\} \in D\right\}$. Since the union of all the $B_{i j}$ is $\omega_{1}$ there must be $i, j$ such 
that $B_{i j} \in D$. In particular, $\left|B_{i j}\right|=\omega_{1}$. Note that there is $\xi$ such that $\xi=\xi_{i}^{\beta}$ for every $\beta \in B_{i j}$, for if $\beta, \gamma \in B_{i j}$ and $\delta$ is such that $z_{\beta}, z_{\delta}$ and $z_{\gamma}, z_{\delta}$ both disagree at $(i, j)$ then $\xi_{i}^{\beta}=\xi_{i}^{\delta}=\xi_{i}^{\gamma}$.

Claim. If $\beta, \gamma \in B_{i j}$ then there is $\delta$ such that $z_{\beta}, z_{\delta}$ and $z_{\gamma}, z_{\delta}$ disagree at $(i, j)$ and $\alpha_{i}^{\beta}, \alpha_{i}^{\gamma}<\alpha_{j}^{\delta}$.

Proof. Suppose not. Then without loss of generality we may assume that $B=\{\delta$ : $z_{\beta}, z_{\delta}$ disagree at $(i, j)$ and $\left.\alpha_{j}^{\delta}<\alpha_{i}^{\beta}\right\} \in D$. Since $\xi_{j}^{\delta}=\xi$ for every $\delta \in B$ we have $\xi_{j}^{\beta}=\xi$. It follows that the $\alpha_{j}^{\delta}$ for $\delta \in B$ are all distinct since otherwise they would all be equal to $\alpha_{j}^{\beta}$ and $z_{\beta}$ would disagree with itself at $(i, j)$, which is impossible. But now if $\delta, \varepsilon \in B$ and $\delta \neq \varepsilon$ we must have $\xi \in g\left(\left\{\alpha_{j}^{\delta}, \alpha_{i}^{\beta}\right\}\right) \cap g\left(\left\{\alpha_{j}^{\varepsilon}, \alpha_{i}^{\beta}\right\}\right)$. Since $f\left(\left\{\alpha_{j}^{\delta}, \alpha_{j}^{\varepsilon}\right\}\right)$ contains either $g\left(\left\{\alpha_{j}^{\delta}, \alpha_{i}^{\beta}\right\}\right)$ or $g\left(\left\{\alpha_{j}^{\varepsilon}, \alpha_{i}^{\beta}\right\}\right)$ we have $\xi \in f\left(\left\{\alpha_{j}^{\delta}, \alpha_{j}^{\varepsilon}\right\}\right)$. Thus if $X=\left\{\alpha_{j}^{\delta}: \delta \in B\right\}$ then $\xi \in \cap f\left([X]^{2}\right)$, contradicting the choice of $f$ and proving the claim.

But now using $\delta$ as in the claim, we may repeat the last part of the proof of the claim to show that if $\beta, \gamma \in B_{i j}$ and $\beta \neq \gamma$ then $\alpha_{i}^{\beta}, \alpha_{i}^{\gamma}$ are distinct and $\xi \in f\left(\left\{\alpha_{i}^{\beta}, \alpha_{i}^{\gamma}\right\}\right)$. This also contradicts the choice of $f$, and proves the theorem.

A consequence of the next result in this section is another characterization very similar to that in the previous theorem. This result, however, may be of some independent interest because of what it says about Chang's conjecture.

THEOREM 2.5. For any ideal I on $\omega_{1}$, the following are equivalent:

(a) $\omega_{2} \rightarrow\left[\omega_{1}\right]_{I^{*}}^{2}$,

(b) $\omega_{2} \rightarrow\left[\omega_{1}: \omega_{1}\right]_{I^{*}}^{2}$; i.e. if $g:\left[\omega_{2}\right]^{2} \rightarrow I^{*}$ then there exist $A, B \in\left[\omega_{2}\right]^{\omega_{1}}$ such that $\cap\{g(\{\alpha, \beta\}): \alpha \in A$ and $\beta \in B\} \neq 0$.

Proof. Only (b) implies (a) requires proof. Suppose then that (b) holds and $f$ : $\left[\omega_{2}\right]^{2} \rightarrow I^{*}$ is given. For each $\beta<\omega_{2}$ let $h_{\beta}: \beta \rightarrow \omega_{1}$ be one-to-one. Now define $g$ : $\left[\omega_{2}\right]^{2} \rightarrow I^{*}$ as follows. If $\alpha<\beta$ then set

$$
g(\{\alpha, \beta\})=\cap\left\{f\left(\left\{\xi_{1}, \xi_{2}\right\}\right): h_{\beta}\left(\xi_{1}\right), h_{\beta}\left(\xi_{2}\right) \leqslant h_{\beta}(\alpha)\right\} .
$$

Notice that $g(\{\alpha, \beta\}) \in I^{*}$ since $I$ is countably complete. Now, since we are assuming (b), there are sets $A, B \subseteq \omega_{1}$ such that both have order-type $\omega_{1}, A \cap B=0$ and $\cap\{g(\{\alpha, \beta\}): \alpha \in A, \beta \in B\} \neq 0$. Choose $\mu$ in this intersection and assume (without loss of generality) that $\sup (B) \geqslant \sup (A)$. To complete the proof, it will suffice to show that $\mu \in \cap f\left([A]^{2}\right)$. So suppose $\left\{\alpha_{1}, \alpha_{2}\right\} \in[A]^{2}$. Choose $\beta \in B$ such that $\beta>\max \left\{\alpha_{1}, \alpha_{2}\right\}$, and assume (without loss of generality) that $h_{\beta}\left(\alpha_{2}\right) \leqslant h_{\beta}\left(\alpha_{1}\right)$. Then $\mu \in g\left(\left\{\alpha_{1}, \beta\right\}\right)$ so $\mu \in f\left(\left\{\alpha_{1}, \alpha_{2}\right\}\right)$ since $h_{\beta}\left(\alpha_{1}\right) \leqslant h_{\beta}\left(\alpha_{1}\right)$ and $h_{\beta}\left(\alpha_{2}\right) \leqslant h_{\beta}\left(\alpha_{1}\right)$.

COROLlaRY 2.6. $\omega_{2} \rightarrow\left[\omega_{1}\right]_{\omega_{1 / \omega}}^{2}$ iff $\omega_{2} \rightarrow\left[\omega_{1}: \omega_{1}\right]_{\omega_{1 / \omega}}^{2}$

Proof. Apply Theorem 2.5 to the ideal $I_{\omega_{1}}$.

We conclude this section with a final set of equivalences involving Martin's Axiom. 
THEOREM 2.7. The following are equivalent:

(1) ZFC + For every $\omega_{2}$-saturated ideal on $\omega_{1}$, there exists a c.c.c. partial ordering $P$ so that $\mathbb{t}_{P}$ "the ideal generated by $I$ is not $\omega_{2}$-saturated".

(2) $\mathrm{ZFC}+M A_{\omega_{2}}+$ There are no $\omega_{2}$-saturated ideals on $\omega_{1}$.

(3) ZFC $+M A+2^{\aleph_{0}}=\boldsymbol{\aleph}_{3}+$ There are no $\omega_{2}$-saturated ideals on $\omega_{1}$.

Proof. (1) $\rightarrow(2)$. Assume that (1) holds, $M A_{\omega_{2}}$ holds, and $I$ is an $\omega_{2}$-saturated ideal on $\omega_{1}$. We seek a contradiction. Choose $P$ (by (1)) so that $\vdash_{P}$ "The ideal generated by $I$ is not $\omega_{2}$-saturated". Then by Theorem 2.2 we know that $T H(I \mid A, P)$ holds for some $A \in I^{+}$. Without loss of generality, assume that $A=\omega_{1}$ and, thus, $T H(I, P)$ holds. Now it is easy to see that we can get a subordering $P^{\prime}$ of $P$ so that $\left|P^{\prime}\right|=\omega_{2}$ and $T H\left(I, P^{\prime}\right)$ holds also. Using $M A_{\omega_{2}}$ it is now easy to show that $P^{\prime}$ is $\sigma$-centered.

Looking ahead to Theorem 3.1 we see that $\vdash_{P^{\prime}}$ " $I$ generates an $\omega_{2}$-saturated ideal on $\omega_{1}$ ". Hence, by Theorem 2.2 again, $T H\left(I, P^{\prime}\right)$ fails. This is the desired contradiction.

$(2) \rightarrow(3)$. Trivial.

(3) $\rightarrow$ (1). Suppose that (1) fails. Then there is a model $V$ of ZFC containing an $\omega_{2}$-saturated ideal $I$ on $\omega_{1}$ having the property that $I$ generates an $\omega_{2}$-saturated ideal in any c.c.c. generic extension of $V$. By Theorem 2.4 it follows that $I$ satisfies Chang's conjecture. Now, it is easy to see that Chang's conjecture for an ideal is preserved when forcing with an $\omega_{2}$-complete partial ordering (i.e. the ideal $\bar{I}$ generated by $I$ in the extension will also satisfy Chang's conjecture). Hence, we can extend $V$ to $V^{\prime}$ by collapsing $2^{\omega_{2}}$ to $\omega_{3}$ via conditions of size $\omega_{2}$ and we will have, in $V^{\prime}$, an $\omega_{2}$-saturated ideal $I^{\prime}$ which generates an $\omega_{2}$-saturated ideal in any c.c.c. generic extension of $V^{\prime}$ (this uses Theorem 2.4 again). But now we can do a c.c.c. iteration over $V^{\prime}$ of length $\omega_{3}$ to obtain a model contradicting (3).

3. Preservation of $\omega_{2}$-saturation. Some preservation (and nonpreservation) results follow rather easily from the equivalents obtained in $\$ 2$. For example, one immediately obtains the following.

THEOREM 3.1. If $I$ is an $\omega_{2}$-saturated ideal on $\omega_{1}$ and $P$ has the $\sigma$-finite chain condition, then

$$
\vdash_{P} \text { "I generates an } \omega_{2} \text {-saturated ideal on } \omega_{1} " .
$$

Proof. Let $U$ be $B(I)$-generic over $V$. Since $j$ is elementary, $j(P)$ has the $\sigma$-finite c.c. in $V^{\omega_{1}} / U$. But then clearly $j(P)$ has the $\sigma$-finite c.c., hence the c.c.c., in $V[U]$.

COROLlARY 3.2. If the existence of a huge cardinal is consistent with $\mathrm{ZFC}$, then $\mathrm{CH}$ is consistent with and independent of the existence of an $\omega_{2}$-saturated ideal on $\omega_{1}$.

The consistency half of Corollary 3.2 is due to Kunen, since $\mathrm{CH}$ holds in his original model $\left[\mathbf{K u}_{2}\right]$ containing an $\omega_{2}$-saturated ideal on $\omega_{1}$. The independence follows from Theorem 3.1 since the orderings for adding (say) Cohen reals and random reals satisfy the $\sigma$-finite chain condition. The independence was noticed 
independently by Richard Laver and a similar result follows immediately from Van Wesep's observation that $2^{\boldsymbol{N}_{0}}=2^{\boldsymbol{N}_{1}}$ in his model [VW] where he gets the $\omega_{2}$-saturation of $N S_{\omega_{1}}$ (but starting with a model of $A D$ etc. instead of "just" a huge cardinal).

It also turns out that the usual c.c.c. partial ordering for forcing $\sigma$-linked $M A$ has the $\sigma$-finite chain condition. Moreover, $\sigma$-linked $M A$ implies the well-known combinatorial property $P(c)$ : if $\kappa<2^{\kappa_{0}}$ and $\left\{X_{\alpha}: \alpha<\kappa\right\} \subseteq \mathscr{P}(\omega)$ is such that finite intersections of the $X_{\alpha}$ 's are infinite, then there is $X \in[\omega]^{\omega}$ such that $X-X_{\alpha}$ is finite for each $\alpha<\kappa$. Hence, assuming the consistency of an $\omega_{2}$-saturated ideal on $\omega_{1}$, we obtain the following.

COROllary 3.3. o-linked $M A$ (and hence $P(c)$ ) is consistent with $2^{\aleph_{0}}>\aleph_{1}$ and the existence of an $\omega_{2}$-saturated ideal on $\omega_{1}$.

In fact, as was remarked by the referee, Corollary 3.3 may be extended to include $M A$ for partial orderings with the $\sigma$-finite chain condition, since if $P$ is any finite support iteration of orderings with the $\sigma$-finite chain condition then $j(P)$ has the same property in $V^{\omega_{1}} / U$. An easy argument now shows that $j(P)$ has the c.c.c. in $V[U]$.

It should be emphasized that we have been unable to find any c.c.c. partial ordering which fails to satisfy Theorem 2.2 (c), so it is conceivable that $\omega_{2}$-saturation is always preserved by c.c.c. orderings. One of the most likely candidates for a counterexample is the ordering for adding a generic path through a Souslin tree. If the Souslin tree $T$ is obtained generically with finite conditions (see $[\mathbf{J}]$; the construction is due to Tennenbaum, but amounts to adding $\omega_{1}$ Cohen reals), then we can show that for any $\omega_{2}$-saturated ideal on $V$, its canonical extension in $V[T]$ remains $\omega_{2}$-saturated after adding a generic path $G$ through $T$. Essentially, the reason is that both $V[T]$ and $B[T, G]$ are $\sigma$-centered extensions of $F$. Details are left to the reader.

Our final two results in this section are in part motivated by our consideration of $\sigma$-finite c.c. orderings in [BT]. The situation that occurred there was the following. We showed that if one forces over a model of $\mathrm{GCH}$ with a $\sigma$-finite c.c. ordering, then in the extension every ideal on $\omega_{1}$ is $\omega_{3}$-saturated. There, however, we were able to sharpen this result considerably. That is, " $\sigma$-finite c.c." could be replaced by "cardinal-preserving $\omega_{1}$-finite c.c." but not by "c.c.c.". This latter observation used a variant of a partial ordering invented by Galvin and Hajnal. It turns out that the situation for preservation of $\omega_{2}$-saturation is somewhat different, both for cardinal preserving $\omega_{1}$-finite c.c. orderings and for the Galvin-Hajnal ordering; this is the content of the following two results.

THEOREM 3.4. If $P$ is the variant of the Galvin-Hajnal ordering used in $\S 6$ of [BT], then the $\omega_{2}$-saturation of any ideal on $\omega_{1}$ is preserved when forcing with $P$.

Proof. We need only show that $P$ satisfies condition (c) of Theorem 2.2, and for this we assume that the reader is familiar with the construction of $P$ in [BT]. Now, since $P$ is built up from the graphs $G_{\alpha}=\left(\kappa, E_{\alpha}\right)$ the same is true of $j(P)$ in $V^{\omega_{1}} / U$. 
But the crucial property of the graphs $G_{\alpha}$ (namely that for any $\xi<\kappa,\{\eta<\xi$ : $\left.\{\eta, \xi\} \in E_{\alpha}\right\}$ has order-type at most $\alpha$ ) requires only countable sets of ordinals for its statement, and it is well known that $V^{\omega_{1}} / U$ and $V[U]$ have the same countable sets of ordinals. Hence $j(P)$ has the c.c.c. in $V[U]$.

Finally, we show that " $\sigma$-finite c.c." cannot be replaced by "cardinal preserving $\omega_{1}$-finite c.c." in Theorem 3.1. We should remark here, however, that our real interest in the next result stems primarily from the considerations to come in $\S \S 4$ and 5 .

THEOREM 3.5. Let $P$ be the partial ordering for adding a closed unbounded subset of $\omega_{1}$ with finite conditions. Then $|P|=\omega_{1}$ (and so $P$ has the $\omega_{1}$-finite c.c.), $P$ is cardinal-preserving (in fact "proper"), and

$$
\mathfrak{}_{P} \text { "There are no } \omega_{2} \text {-saturated ideals on } \omega_{1} \text { ". }
$$

Proof. Suppose that $G$ is $P$-generic over $V$ and assume, for contradiction, that $I$ is an $\omega_{2}$-saturated ideal in $V[G]$. Let $U$ be $B(I)$-generic over $V[G]$ and let $j: V[G] \rightarrow N$ be the associated elementary embedding. Now, working in $V[G][U]$, let $M=$ $\cup\left\{j\left(V_{\alpha}\right): \alpha \in O R\right\}$. Since $j$ is an elementary embedding, it follows that $N=$ $M[j(G)]$ where $j(G)$ is $j(P)$-generic over $M$.

Now, it is easy to see that $j(P)$ is just the partial ordering for adding a closed unbounded subset of $\omega_{2}^{V}$ by finite conditions. Hence, $j(P)$ is an element of $V$.

Since $j\left(\omega_{1}\right)=\omega_{2}$ we see that $j(G)$ gives us, in $M[j(G)]$, a closed unbounded set $C \subseteq \omega_{1}^{M[j(G)]}=\omega_{2}^{M}$. Now, because $I$ is $\omega_{2}$-saturated we know that $V[G][U]$ and $M[j(G)]$ have the same countable sets of ordinals and so $C$ is a closed unbounded subset of $\omega_{1}^{V[G][U]}=\omega_{2}^{V}$ in $V[G][U]$. Now notice that $V[G][U]$ is an $\omega_{2}^{V}$-c.c. extension of $V$ (since $|P|=\omega_{1}$ and $B(I)$ is $\omega_{2}$-saturated), and so there is a closed unbounded set $C_{1} \in V$ such that $C_{1} \subseteq C$.

By the fundamental property of adding a closed unbounded set with finite conditions, we know that no countable subset of $C$ is an element of $M_{j}$ and so, $a$ fortiori, no countable subset of $C_{1}$ is an element of $M$. In view of this, the desired contradiction is an immediate consequence of the following claim.

Claim. Suppose that $j: V \rightarrow M$ is an elementary embedding such that $j \mid \omega_{1}$ is the identity and $j\left(\omega_{1}\right) \geqslant \omega_{2}$. Then for any bounded subset $X \subseteq \omega_{2}^{V}$, if $X \in V$ then $X \in M$.

Proof. If $X \subseteq \omega_{1}^{V}$, then $X=j(X) \cap \omega_{1} \in M$. If $\alpha<\omega_{2}^{V}$ and $X \subseteq \alpha$, then there is $Y \subseteq \omega_{1}^{V}$ such that $Y \in V$ and $X \in L[Y]$. Then $Y \in M$, so $X \in M$ also.

4. Basic properties of presaturated ideals. In this section we consider a class of ideals on $\omega_{1}$ characterized by a property that lies (strictly) between the notions of precipitousness and $\omega_{2}$-saturation. This fact suggests the term "presaturated" for ideals in this class.

DEFINITION 4.1. If $I$ is an ideal on $\omega_{1}$, then $I$ will be called

(a) $\omega_{2}$-preserving iff $t_{B(I)}$ " $\omega_{2}^{V}$ is a cardinal".

(b) presaturated iff $I$ is precipitous and $\omega_{2}$-preserving. 
It is, of course, well known that an $\omega_{2}$-saturated ideal on $\omega_{1}$ is both precipitous and $\omega_{2}$-preserving; hence, $\omega_{2}$-saturated $\Rightarrow$ presaturated $\Rightarrow$ precipitous. It will be shown in $\$ 5$ that these implications are nonreversible (in a rather strong sense). Here, however, we concentrate on some structural properties of presaturated ideals, beginning with the following combinatorial equivalent of Definition 4.1(a).

THEOREM 4.2. For an $\omega_{3}$-saturated ideal on $\omega_{1}$, the following are equivalent.

(a) I is $\omega_{2}$-preserving.

(b) $\vdash_{B(I)} " \operatorname{cf}\left(\omega_{2}^{V}\right) \neq \omega "$.

(c) If for each $n \in \omega$ we have that $\left\{X_{\alpha}^{n}: \alpha<\omega_{2}\right\}$ is a set of pairwise I-almost disjoint elements of $I^{+}$, then for every $A \in I^{+}$there is $B \in \mathscr{P}(A) \cap I^{+}$so that $\mid\left\{\alpha<\omega_{2}\right.$ : $\left.X_{\alpha}^{n} \cap B \in I^{+}\right\} \mid \leqslant \omega_{1}$ for each $n \in \omega$.

Proof. (a) $\rightarrow$ (b). This is obvious.

(b) $\rightarrow$ (c). Suppose (b) holds and that for each $n \in \omega$ we have $\left\{X_{\alpha}^{n}: \alpha<\omega_{2}\right\}$ as in (c). Define $\dot{f}: \omega \rightarrow \omega_{2}$ so that $\left[X_{\alpha}^{n}\right] \Vdash \dot{f}(n)=\alpha$. By (b), we know that $\vdash_{B(I)} "(\exists \alpha<$ $\left.\omega_{2}\right)(\forall n \in \omega)(\dot{f}(n)<\alpha)$ ". Now suppose $A \in I^{+}$is given. Then there is $B \in \mathscr{P}(A) \cap$ $I^{\dagger}$ and $\alpha<\omega_{2}$ such that $[B] \mid{ }^{\prime \prime} "(\forall n \in \omega)(\dot{f}(n)<\alpha)$ ". But now it is easy to see that if $X_{\beta}^{n} \cap B \in I^{+}$then $\beta<\alpha$, and so $B$ shows that (c) holds.

(c) $\rightarrow$ (a). Suppose $I$ is not $\omega_{2}$-preserving. Then for some $A \in I^{+}$we have that $[A]$ it $\left|\omega_{2}^{V}\right|=\omega$. This is a consequence of the observation (see Proposition III.4.1 of [BTW]) that $\omega_{1}$ is always collapsed when forcing with $B(I)$ where $I$ is any ideal on $\omega_{1}$. So let $\dot{f}$ be a term such that $[A] \Vdash \dot{f}: \omega \stackrel{\text { onto }}{\rightarrow} \omega_{2}$. For each $n$ let $P_{n}$ be a maximal family of subsets of $A$ whose equivalence classes in $B(I)$ force different values for $\dot{f}(n)$. Infinitely many of the $P_{n}$ 's must have size $\omega_{2}$, so, for simplicity, assume they all do. But now if $B \in \mathcal{P}(A) \cap I^{+}$and $B$ hits only $\omega_{1}$ of the sets in each $P_{n}$ in a set of positive $I$-measure, then clearly $[B] \mathbb{F}^{\text {" }} \dot{f}$ is bounded". This contradiction completes the proof.

It may be that the requirement of precipitousness in the definition of presaturated is redundant. That is, it seems plausible that every $\omega_{2}$-preserving ideal on $\omega_{1}$ might of necessity be precipitous, although we are unable to prove this. We do, however, have the following partial result along these lines.

THEOREM 4.3. Suppose that I is an $\omega_{2}$-preserving ideal on $\omega_{1}$ that is $\omega_{\omega}$-saturated and is such that for every $m<\omega, \mathfrak{}_{B(I)} \operatorname{cf}\left(\omega_{m}^{V}\right) \neq \omega$. Then $I$ is precipitous and hence presaturated.

Proof. Suppose that $I$ is a counterexample, and let $\kappa$ be the least cardinal for which $I$ is $\kappa$-saturated. Then $\kappa \leqslant \omega_{\omega}$ and it is well known that $\kappa$ must be regular; hence $\kappa=\omega_{n}$ for some $n$ with $2 \leqslant n<\omega$. By assumption, there is a set $A \in I^{+}$so that $[A] \mid \vdash_{B(I)}$ " $\left\langle\left[\dot{f}_{j}\right]: j \in \omega\right\rangle$ is a descending sequence in the generic ultrapower".

Now, for each $A \in I^{+}$and each such sequence we can choose, for $j \in \omega$, a maximal family $\left\{X_{\alpha}^{j}: \alpha<\lambda_{j}\right\} \subseteq I^{+}$of $I$-almost disjoint subsets of $A$ so that for some $f_{\alpha}^{j} \in V$ we have $\left[X_{\alpha}^{j}\right] \Vdash \dot{f}_{i}=f_{\alpha}^{j}$. It is easy to see that such a family is really a maximal $I$-almost disjoint family in $(I \mid A)^{+}$, and so for each $j$ we have $\lambda_{j} \leqslant \omega_{n}$. Without loss 
of generality, assume that $\lambda_{j}=\omega_{m}$ for every $j<\omega$, and among all such sets $A \in I^{+}$ and all such sequences that we have chosen the ones yielding the smallest possible value for $m$.

We claim now that $m \leqslant 1$. Suppose not. Let $\dot{f}$ be a term defined so that for $j \in \omega$ and $\alpha \in \omega_{m}$ we have $\left[X_{\alpha}^{j}\right] \Vdash \dot{f}(j)=\alpha$. Then $[A] \Vdash$ "domain $(\dot{f}) \subseteq \omega$ ", and so, since $I \mid A$ satisfies the same conditions imposed on $I$, we have some set $B \in(I \mid A)^{+}$and some ordinal $\gamma<\omega_{m}$ so that $[B] \Vdash F^{\prime}$ range $(\dot{f}) \subseteq \gamma$ ". But now it is easy to see that the sequences $\left\{X_{\alpha}^{j} \cap B: \alpha<\gamma, j<\omega\right\}$ contradict our choice of $A$, thus showing that $m \leqslant 1$ as desired.

To complete the proof, we need only note now that since $m \leqslant 1$, it is easy to find a function $g_{j} \in V$ so that $\operatorname{domain}\left(g_{j}\right)=A$ and $[A] \Vdash \dot{f}_{j}=g_{j}$. Then, except for a set in $I$, we have that for every $\xi \in A, g_{j+1}(\xi)<g_{j}(\xi)$; contradiction.

A version of Theorem 4.3 is implicit in the work of Balcar and Franek [BF]. They show there that if $I$ is nowhere precipitous (i.e. no $I \mid A$ is precipitous) and if $B(I)$ has a dense set of size $\omega_{2}$, then $B(I)$ is isomorphic to the usual Levy collapse of $\omega_{2}$ to $\omega$. It turns out that if $I$ has a dense set of size $\omega_{2}$, then $I$ fails to be presaturated iff $B(I \mid A)$ is isomorphic to this Levy collapse for some $A \in I^{+}$. In fact, let $\operatorname{Col}(\omega, \kappa)$ be the complete Boolean algebra corresponding to adding a surjection $f: \omega \rightarrow \kappa$ by finite conditions, let $B$ be a complete atomless Boolean algebra and let $\kappa$ be a regular uncountable cardinal such that $t_{B} \operatorname{cf}(\kappa)=\omega$. Then $B \cong \operatorname{Col}(\omega, \kappa)$ iff $B$ has a dense set of size $\kappa$. The proofs are left for the reader.

The last two results in this section require some concepts from the structural theory of ideals (e.g. see [BTW]). First of all, if $I$ is an ideal on $\kappa, A \in I^{+}$and $f$ : $A \rightarrow \kappa$, then $f$ is called I-small iff $f^{-1}(\{\alpha\}) \in I$ for every $\alpha<\kappa$, and in this case $f_{*}(I)$, which denotes $\left\{X \subseteq \kappa: f^{-1}(X) \in I\right\}$, is an ideal on $\kappa$. The Rudin-Keisler (pre-) ordering $\leqslant_{R K}$ of ideals on $\kappa$ is defined by $J \leqslant_{R K} I$ iff $J=f_{*}(I)$ for some $I$-small $f: \kappa \rightarrow \kappa$. Two ideals $I$ and $J$ on $\kappa$ are said to be isomorphic (denoted $I \cong J$ ) iff $J=f_{*}(I)$ for some bijection $f: \kappa \rightarrow \kappa$.

An ideal $I$ on $\kappa$ is said to be a P-point (local P-point) iff for every $I$-small $f: \kappa \rightarrow \kappa$ there exists a set $X \in I^{*}\left(X \in I^{+}\right)$such that $f \mid X$ is less than $\kappa$ to one. $I$ is a weak $P$-point iff $I \mid A$ is a local $P$-point for every $A \in I^{+}$. (Hence, $P$-point $\Rightarrow$ weak $P$-point $\Rightarrow$ local $P$-point.)

It is known $\left[\mathbf{T}_{1}\right]$ that if $I$ is an $\omega_{2}$-saturated ideal on $\omega_{1}$, then $I$ is a $P$-point. This fails badly for precipitous ideals, and there can even be precipitous ideals on $\omega_{1}$ that are not weak $P$-points. For presaturated ideals, however, we have the following.

THEOREM 4.4. If I is an $\omega_{2}$-preserving ideal on $\omega_{1}$, then I is a weak P-point.

Proof. Suppose that $I$ is not a weak $P$-point. Then for some $A \in I^{+}$, the ideal $I \mid A$ is not a local $P$-point. Since an ideal is $\omega_{2}$-preserving iff every restriction of it is $\omega_{2}$-preserving, we lose no generality in assuming that $A=\omega_{1}$, and hence $I$ is not a local $P$-point. Now Theorem III.1.1 and Corollary III.4.11 of [BTW] show that $T H(I)$ holds whenever $I$ is an ideal on $\omega_{1}$ that fails to be a local $P$-point. That is, there exists a family $\left\{g_{\alpha}: \alpha<\omega_{2}\right\}$ of functions mapping $\omega_{1}$ to $\omega$ such that if $\alpha<\beta<\omega_{2}$ then $A_{\alpha \beta}=\left\{\xi<\omega_{1}: g_{\alpha}(\xi)=g_{\beta}(\xi)\right\} \in I$. 
Now let $U$ be $B(I)$-generic over $V$ and consider the generic ultrapower $V^{\omega_{1}} / U$. For $\alpha<\omega_{2}$, each $\left[g_{\alpha}\right]$ represents an element of $\omega$ in $V^{\omega_{1}} / U$ and for $\alpha \neq \beta$ we have $\left[g_{\alpha}\right] \neq\left[g_{\beta}\right]$. Thus, in $V[U],\left|\omega_{2}^{V}\right|=\omega$ and hence $I$ is not $\omega_{2}$-preserving.

Solovay proved $[\mathbf{S}]$ that if there is an $\omega_{2}$-saturated ideal $I$ on $\omega_{1}$ then there is a normal $\omega_{2}$-saturated ideal $J$ on $\omega_{1}$, and in fact $J$ can be taken to be below $I$ in the Rudin-Keisler ordering of ideals on $\omega_{1}$. It is not known if the existence of a precipitous ideal on $\omega_{1}$ implies the existence of a normal precipitous ideal on $\omega_{1}$, but for presaturated ideals we have the following.

THEOREM 4.5. If there is a presaturated ideal I on $\omega_{1}$, then there is a normal presaturated ideal $J$ on $\omega_{1}$.

Proof. We will show that if $I$ is presaturated, then $I$ is, in fact, "densely isomorphic" to a normal ideal. That is, for every $A \in I^{+}$there is some $B \in \mathscr{P}(A) \cap$ $I^{+}$such that $I \mid B$ is isomorphic to a normal ideal (which must then also be presaturated). So suppose that $A \in I^{+}$is given. Then, since $I$ is precipitous, Theorem III.3.4 of [BTW] guarantees that for some $B \in \mathscr{P}(A) \cap I^{+}$we have that $I \mid B$ is isomorphic to some ideal $J$ extending $N S_{\omega_{1}}$. But $I \mid B$ (and hence $J$ ) is a weak $P$-point by Theorem 4.4 and so the desired result now follows from the observation (Lemma III.1.11 of [BTW]) that any weak $P$-point extension of $N S_{\omega_{1}}$ is a normal ideal.

Notice that the above proof does not yield a normal presaturated ideal $J$ that is $R K$ below the given presaturated ideal $I$. We do not know if Theorem 4.5 can be strengthened to yield this.

5. Further preservation results. In this section we will extend Theorem 2.2 to cover a larger class of ideals, including the precipitous and presaturated ideals, as well as a larger class of partial orderings.

Enlarging the class of orderings, however, leads to the following difficulty. Let $I$ be a countably complete ideal on $\omega_{1}$ and let $P$ be a partial ordering. If $G$ is $P$-generic then in $V[G]$ we may form the ideal $\bar{I}=\left\{A \subseteq \omega_{1}^{V}: \exists B \in I(A \subseteq B)\right\}$. Now if $P$ has the countable chain condition, then $\bar{I}$ will be countably complete, but this is not true for arbitrary $P$. Since we are only interested in countably complete ideals, we need a condition on $P$ to guarantee the completeness of $\bar{I}$.

Perhaps the most natural such condition is

(*) if $p \in P$ and $p \vdash^{\prime \prime} \dot{a}$ is a countable subset of $V$ ", then there is countable $b \in V$ and $q \leqslant p$ such that $q \Vdash \dot{a} \subseteq b$.

Every proper partial ordering satisfies $(*)$, and $(*)$ clearly implies that forcing with $P$ preserves $\omega_{1}$.

Although we will not use it here, there is another way to handle the difficulty above which might be mentioned. Instead of using $\bar{I}$ one can simply use the $\sigma$-ideal $J$ generated by $I$ in $V[G]$. Of course, if $P$ collapses $\omega_{1}$, then $J$ is not even a proper ideal, but this raises the following question, which we have been unable to answer. If $P$ preserves $\omega_{1}$, does it follow that $J$ is always a proper ideal?

Throughout the rest of this section, $I$ is a countably complete ideal on $\omega_{1}, U$ is $B(I)$-generic (over $V$ ), $M$ is the ultrapower $V^{\omega_{1}} / U$ (formed in $V[U]$ ) and $j: V \rightarrow M$ 
is the canonical elementary embedding. If $I$ is precipitous then $M$ is well founded, and in this case we identify $M$ with its transitive collapse. We will freely confuse $U$, $M$ and $j$ with the names for them in the language of forcing with respect to $B(I)$.

Definition 5.1. (a) If $P$ is a partial ordering and $q \in j(P)$, then $P$ is regular below $q$ iff for any dense $D \subseteq P, D \in V$, and any $r \leqslant q$, there is $p \in D$ such that $j(p)$ and $r$ are compatible (in $j(P)$ ).

(b) $P$ is $I$-regular iff $\vdash_{B(I)} \forall p \in P \exists q \in j(P)(q \leqslant j(p)$ and $P$ is regular below $q)$.

Note that $P$ is regular below $q$ iff in $V[U], q \vdash_{j(P)}$ " $j^{-1}(\dot{H})$ is $P$-generic over $V$ ", where $\dot{H}$ is the canonical name for the set $j(P)$-generic over $V[U]$.

It is not hard to see that if $P$ has the countable chain condition, then $P$ is $I$-regular for all $I$; this will be verified in the course of proving Theorem 5.7 below. A consequence of Theorem 5.8 below is that if $P$ is the ordering for adding a closed unbounded subset of $\omega_{1}$ with finite conditions, then $P$ is $I$-regular for every precipitous ideal $I$. Moreover, it is consistent that the Cohen ordering for adding a subset of $\omega_{1}$ with countable conditions is $I$-regular for certain $I$. The same applies to the Sacks ordering and to Axiom A orderings of cardinality $\leqslant 2^{\aleph_{0}}$ in general. There are more complete remarks following Lemma 5.13 below.

Now suppose $P$ satisfies (*) and $H$ is $j(P)$-generic over $V[U]$. Let $G=j^{-1}(H)$. Suppose also that $q \in H$ and $P$ is regular below $q$. Then $G$ is $P$-generic over $V$. Let $\bar{I}$ be the ideal in $V[G]$ generated by $I$, and let $\bar{U}=\left\{A \in \bar{I}^{+}:\right.$if $A=\dot{A}^{V[G]}$ then $\exists r \in H(M \vdash$ " $r \vdash[$ id] $\in j(\dot{A})$ ") $\}$. Here [id] denotes the equivalence class of the identity function on $\omega_{1}$ in the ultrapower $M$. It is not difficult to see that $\bar{U}$ is an ultrafilter in $\mathcal{P}\left(\omega_{1}\right) \cap V[G]$, although of course $\bar{U} \in V[U][H]$. It turns out that even more is true.

THEOREM 5.2. $\bar{U}$ is $B(\bar{I})$-generic over $V[G]$. Moreover, if $I$ is precipitous then $j$ : $V \rightarrow M$ may be extended to $\bar{j}: V[G] \rightarrow M[H]$, and $\bar{U}=\left\{A \subseteq \omega_{1}:[\mathrm{id}] \in \bar{j}(A)\right\}$.

Proof. Without loss of generality, we may assume that $P$ is a dense subset of a complete Boolean algebra $B$. If $X \in I^{+}$and $f: X \rightarrow B$ then let $\operatorname{ess} \sup (f)=$ $\wedge_{C \in I^{*}}\left(\bigvee_{\alpha \in X \cap C} f(\alpha)\right)$.

Lemma 5.3. Suppose $X \in I^{+}, f: \omega_{1} \rightarrow P$ and $[X] \Vdash P$ is regular below $[f]$. Then ess $\sup (f \mid X) \neq 0$.

Proof. Suppose ess sup $(f \mid X)=0$. Then

$$
D=\left\{p \in P:\left(\exists C \in I^{*}\right) p \wedge\left(\vee_{\alpha \in X \cap C} f(\alpha)\right)=0\right\}
$$

is dense in $P$. But if $C \in I^{*}$ and $p \wedge\left(\vee_{\alpha \in X \cap C} f(\alpha)\right)=0$, then clearly

$$
[X] \Vdash j(p) \text { and }[f] \text { are incompatible. }
$$

But this contradicts the assumption that

$$
[X] \Vdash P \text { is regular below }[f] \text {. }
$$

If $\Phi$ is a sentence of the language of forcing with respect to $P$, then $\|\Phi\|$ will represent the Boolean value (in $B$ ) of $\Phi$. If $f: \omega_{1} \rightarrow B$ then clearly there is a term $\dot{X}(f)$ such that for every $\alpha<\omega_{1},\|\alpha \in \dot{X}(f)\|=f(\alpha)$. 
Lemma 5.4. If $f: \omega_{1} \rightarrow B$ then $p \Vdash \dot{X}(f) \in \bar{I}^{+}$iff $p \leqslant \operatorname{ess} \sup (f)$.

Proof. Suppose $q \leqslant p \leqslant \operatorname{ess} \sup (f)$. Let $C \in I^{*}$. Then there is some $\alpha \in C$ so that $q \wedge f(\alpha) \neq 0$. Choose $r \leqslant q \wedge f(\alpha)$. Then $r \Vdash \dot{X}(f) \cap C \neq 0$, so $p \Vdash \forall C \in$ $I^{*}(\dot{X}(f) \cap C \neq 0)$, i.e., $p \Vdash \dot{X}(f) \in \bar{I}^{+}$.

Suppose $p \neq \operatorname{ess} \sup (f)$. Then there is $C \in I^{*}$ and $q \leqslant p$ such that $q \wedge$ $\left(\vee_{\alpha \in C} f(\alpha)\right)=0$. But clearly $q \Vdash \dot{X}(f) \subseteq \omega_{1}-C$, so $p \Vdash \dot{X}(f) \in \bar{I}^{+}$.

Now we return to Theorem 5.2. All we need prove is that if $\left\langle X_{\xi}: \xi<\lambda\right\rangle$ is a maximal sequence of $\bar{I}$-almost disjoint elements of $\bar{I}^{+}$(i.e., the intersection of distinct elements lies in $\bar{I}$ ), then $X_{\xi} \in \bar{U}$ for some $\xi<\lambda$.

Let $\left\langle\dot{X}_{\xi}: \xi<\lambda\right\rangle$ be a term denoting $\left\langle X_{\xi}: \xi<\lambda\right\rangle$ and let $p \in G$ be such that

$p \vdash\left\langle\dot{X}_{\xi}: \xi<\lambda\right\rangle$ is a maximal $\bar{I}$-almost disjoint sequence from $\bar{I}^{+}$.

Let $f_{\xi}(\alpha)=p \wedge\left\|\alpha \in \dot{X}_{\xi}\right\|$. Note that $p \Vdash \dot{X}\left(f_{\xi}\right)=\dot{X}_{\xi}$.

Since $p \in G$ we have $j(p) \in H$. Hence there must be $q^{\prime} \leqslant q, j(p)$ with $q^{\prime} \in H$. Then $P$ is regular below $q^{\prime}$ also. Let $E=\left\{r \leqslant q^{\prime}: \exists \xi\left(r \leqslant\left[f_{\xi}\right]\right)\right\}$.

Lemma 5.5. $E$ is dense below $q^{\prime}$ in $j(P)$.

Proof. If not then there is $r \leqslant q^{\prime}$ which is incompatible with all $\left[f_{\xi}\right]$. Say $r=[f]$. There must be $X \in U$ such that

$[X] \mid{ }^{\prime}$ " $P$ is regular below $[f],[f]$ is incompatible with all $\left[f_{\xi}\right]$, and $[f] \leqslant j(p)$ ".

By Lemma 5.3, ess sup $(f \mid X) \neq 0$, and clearly ess $\sup (f \mid X) \leqslant p$. If $p^{\prime} \in P$ and $p^{\prime} \leqslant \operatorname{ess} \sup (f \mid X)$ then by Lemma 5.4, $p^{\prime} \mid r$ “ $\dot{X}(g) \in \bar{I}^{+}$”, where $g|X=f| X$ and $g$ is 0 on $\omega_{1}-X$. Since $\left.p^{\prime}\right|^{\prime \prime} "\left\langle X_{\xi}: \xi<\lambda\right\rangle$ is maximal", there is $p^{\prime \prime} \leqslant p^{\prime}$ and $\xi$ so that $p^{\prime \prime} \Vdash \dot{X}_{\xi} \cap \dot{X}(g) \in \bar{I}^{+}$. But then if $Y=\{\alpha \in X: f(\alpha)(=g(\alpha))$ is compatible with $\left.f_{\xi}(\alpha)\right\}$, we must have $Y \in I^{+}$. The same argument would work if $X$ were replaced by any $X^{\prime} \subseteq X$ with $X^{\prime} \in I^{+}$. Thus there must be a $Y$ as above in $U$. But then $[Y] \mid{ }^{\prime}$ “ $\left[f_{\xi}\right]$ and $[f]$ are compatible", contradiction. Hence $E$ is dense below $q^{\prime}$.

Thus, there exists $\xi$ such that $\left[f_{\xi}\right] \in H$. We claim that $X_{\xi} \in \bar{U}$, which will complete the proof of Theorem 5.2. But this is easy, since for every $\alpha$ we have that $f_{\xi}(\alpha) \Vdash \alpha \in \dot{X}_{\xi}$, so $M \vDash "\left[f_{\xi}\right] \Vdash[$ id $] \in j\left(\dot{X}_{\xi}\right)$ ", and $X_{\xi} \in \bar{U}$.

If now $I$ is precipitous then we may define $\bar{j}: V[G] \rightarrow M[H]$ by $\bar{j}^{\left(\dot{X}^{V[G]}\right)=}$ $j(\dot{X})^{M[H]}$. Since $p \in G$ iff $j(p) \in H$ it is straightforward to see that $j$ is elementary and extends $j$. Finally, using the definition of $\bar{j}$ above, it is clear that $\bar{U}=\left\{A \subseteq \omega_{1}\right.$ : [id] $\in \bar{j}(A)\}$.

DEFINITION 5.6. A countably complete ideal $I$ on $\omega_{1}$ is strong iff $I$ is precipitous and $r_{B(I)} j\left(\omega_{1}^{V}\right)=\omega_{2}^{V}$.

It is easy to see that every presaturated ideal is strong. Moreover, in Kunen's proof in $\left[\mathbf{K u}_{\mathbf{1}}\right]$ that the existence of an $\omega_{2}$-saturated ideal on $\omega_{1}$ implies the consistency of the existence of many measurable cardinals, only the fact that the ideal was strong was required. Since the existence of a precipitous ideal is equiconsistent with the existence of a single measurable cardinal [JMMP], it follows that precipitous does not imply strong. 
One reason for introducing strong ideals is that they occupy a natural place in Theorem 5.7 below. Unfortunately we know rather little about them. It is conceivable that strong ideals coincide with presaturated ideals, although we doubt it. On the other hand we conjecture that the consistency of the existence of a strong ideal is equivalent to the consistency of the existence of an $\omega_{2}$-saturated ideal. We have also been unable to prove that the existence of a strong ideal implies the existence of a normal one.

Theorem 5.7. Let $P$ be I-regular.

(a) If I is precipitous then

$$
{ }_{\mathfrak{r}_{P}} \exists A \in \bar{I}^{+}(\bar{I} \mid A \text { is precipitous }) .
$$

(b) If $I$ is strong and $P$ does not collapse $\omega_{2}$, then

$$
{ }_{\vdash_{P}} \exists A \in \bar{I}^{+}(\bar{I} \mid A \text { is strong }) \text {. }
$$

(c) If I is presaturated and $\vdash_{B(I)}$ “ $j(P)$ does not collapse $\omega_{2}^{V}$, then

$$
{ }{ }_{P} \exists A \in \bar{I}^{+}(\bar{I} \mid A \text { is presaturated }) .
$$

(d) If I is $\omega_{2}$-saturated and $\vdash_{B(I)}$ " $j(P)$ has the c.c.c.", then

$$
{ }_{\mathrm{t}_{P}} \bar{I} \text { is } \omega_{2} \text {-saturated. }
$$

Moreover, in (a), (b) and (c) if $P$ has the countable chain condition then we may take $A=\omega_{1}$.

Proof. (a) By Theorem 5.2, $j: V \rightarrow M$ may be extended to $\bar{j}: V[G] \rightarrow M[H]$. Hence $V[G]^{\omega_{1}} / \bar{U}$ is well founded, so there is $A \in \bar{U}$ such that

$$
[A] \Vdash V[G]^{\omega_{1}} / \bar{U} \text { is well founded. }
$$

But then $\bar{I} \mid A$ is precipitous. Hence there is $p \in G$ such that

$$
p \Vdash \exists A \in \bar{I}^{+}(\bar{I} \mid A \text { is precipitous }) .
$$

But since for any $p \in P$ we can always find $q \leqslant j(p)$ so that $P$ is regular below $q$, we can work below any given $p \in P$ as above. Hence $\vdash_{P} \exists A \in \bar{I}^{+}(\bar{I} \mid A$ is precipitous).

In case $P$ has the countable chain condition, note that $P$ is regular below every element of $j(P)$, for if $D \in V$ is a maximal incompatible set then since $D$ is countable $j(D)=\{j(p): p \in D\}$ and $j(D)$ is maximal incompatible in $j(P)$.

Now suppose $p \vdash_{P} \dot{C} \in \bar{I}^{+}$. We claim there is $q \leqslant p$ such that $q \vdash^{*} \exists A \subseteq \dot{C}\left(A \in \bar{I}^{+}\right.$ and $\bar{I} \mid A$ is precipitous)". This implies immediately that $\vdash_{P} \bar{I}$ is precipitous.

For each $\alpha<\omega_{1}$ let $f(\alpha)=\|\alpha \in \dot{C}\|$. Then $p \leqslant \operatorname{ess} \sup (f)$ by Lemma 5.4. Let $C_{0}=\{\alpha: p \wedge f(\alpha) \neq 0\}$. Then $C_{0} \in I^{+}$and $\left[C_{0}\right] \mid r^{\prime \prime} j(p)$ and $[f]$ are compatible". Now let $U$ be $B(I)$-generic with $C_{0} \in U$ and let $H$ be $j(P)$-generic over $V[U]$ with $q^{\prime} \in H, q^{\prime} \leqslant j(p),[f]$. $P$ is automatically regular below $q^{\prime}$. Now clearly

$$
M \mid \vdash^{\prime \prime}[f] \vdash_{j(P)}[\mathrm{id}] \in j(\dot{C}) ”,
$$

so $\dot{C}^{V[G]} \in \bar{U}$. Now, working as before, we can find $A \leqslant \dot{C}^{V[G]}, A \in \bar{U}$, such that $\bar{I} \mid A$ is precipitous (in $V[G]$ ); hence there is $q \leqslant p, q \in G$, such that

$$
q \Vdash \exists A \subseteq \dot{C}\left(A \in \bar{I}^{+} \text {and } \bar{I} \mid A \text { is precipitous }\right)
$$

as desired. 
(b) is easy since $\omega_{2}^{V[G]}=\omega_{2}^{V}$ and $\bar{j}$ extends $j$.

(c) is also easy since $\omega_{2}^{V}$ remains a cardinal in $V[U][H]$, hence in $V[G][\bar{U}]$.

(d) is part of Theorem 2.2 and is included here only for completeness, since the techniques behind Theorem 2.2 have here been generalized.

Remark. Theorem 5.7(a) extends Kakuda's theorem [K] that if $I$ is a precipitous $\kappa$-complete ideal on an uncountable regular cardinal $\kappa$ and $P$ has the $\kappa$-chain condition, then $t_{P} \bar{I}$ is precipitous, at least for the case $\kappa=\omega_{1}$. In fact it is easy to see that all the results of this section may be straightforwardly generalized to regular uncountable $\kappa$, so we have a genuine extension of Kakuda's result. Details are left to the reader.

We have already seen in the proof of Theorem 5.7(a) that any partial ordering with the countable chain condition is $I$-regular for all $I$. It follows that such orderings preserve precipitous and strong ideals. We do not know whether they always preserve presaturated ideals.

Now we turn to other orderings.

THEOREM 5.8. Let $P$ be the partial ordering for adding a closed unbounded subset of $\omega_{1}$ with finite conditions. If $\vdash_{B(I)} V^{\omega_{1}} / U$ has an $\omega_{1}^{V}$ th element, then $P$ is I-regular.

Proof. We may assume that $\omega_{1}^{V}+1 \subseteq M=V^{\omega_{1}} / U$. Let $q=\left\{\left(\omega_{1}^{V}, \omega_{1}^{V}\right)\right\}$. Since $\omega_{1}^{V}$ is countable in $M$ we have $q \in j(P)$. Also, if $D$ is dense in $P$ then it is easy to see that for any $r \leqslant q$, if $p \in D$ is such that $p \leqslant r \cap\left(\omega_{1}^{V} \times \omega_{1}^{V}\right)$, then $r$ and $p=j(p)$ are compatible. Hence $P$ is $I$-regular.

THEOREM 5.9. Let $P$ be the ordering of Theorem 5.8.

(a) If I is precipitous, then $\Vdash_{P} \exists A \in \bar{I}^{+}(\bar{I} \mid A$ is precipitous $)$.

(b) If $I$ is strong, then $\vdash_{P} \exists A \in \bar{I}^{+}(\bar{I} \mid A$ is strong $)$.

(c) If I is presaturated, then $\vdash_{P} \exists A \in \bar{I}^{+}(\bar{I} \mid A$ is presaturated $)$.

If $I$ is normal, $G$ is $P$-generic and $C=\{\alpha:\{(\alpha, \alpha)\} \in G\}$, then in (a), (b) and (c) we may take $A=C$. Moreover, in this case

$$
\left|r_{P} \bar{I}\right| \dot{C} \text { is normal, }
$$

where $\dot{C}$ is the canonical name for $C$.

Proof. (a) follows immediately from Theorems 5.7(a) and 5.8.

(b) is similar, using the fact that $|P|=\omega_{1}$, so forcing with $P$ preserves $\omega_{2}$.

(c) is also similar, but we must observe that $j(P)$ is simply $P$ as defined in $V[U]$ (or $M$ ); hence $j(P)$ must preserve $\omega_{1}^{V[U]}=\omega_{2}^{V}$.

Now suppose $I$ is normal. Since $C$ is closed unbounded in $\omega_{1}$ we have $\vdash_{P} \dot{C} \in \bar{I}^{+}$. Suppose $p \Vdash \dot{A} \subseteq \dot{C}, \dot{A} \in \bar{I}^{+}$. For each $\alpha<\omega_{1}$ let $f(\alpha)=p \wedge\|\alpha \in \dot{A}\|$. Then $A_{0}=$ $\{\alpha: f(\alpha)$ is compatible with $\{(\alpha, \alpha)\}\} \in I^{+}$since clearly $\|\alpha \in \dot{C}\|=\{(\alpha, \alpha)\}$. As in the proof of Theorem 5.7 we have $\left[A_{0}\right] \mid \vdash$ " $[f]$ and $\left\{\left(\omega_{1}^{V}, \omega_{1}^{V}\right)\right\}$ are compatible" (for if $g(\alpha)=\{(\alpha, \alpha)\}$ then $[g]=\left\{\left(\omega_{1}^{V}, \omega_{1}^{V}\right)\right\}$ by the normality of $\left.I\right)$. Hence if $U$ and $H$ are chosen so that $A_{0} \in U$ and $[f],\left\{\left(\omega_{1}^{V}, \omega_{1}^{V}\right)\right\} \in H$ we may conclude as before that there exists $q \leqslant p$ such that $q \Vdash$ " $\exists B \subseteq \dot{A}\left(B \in \bar{I}^{+}\right.$and $\bar{I} \mid B$ is precipitous)". Hence $\left|\vdash_{P} \bar{I}\right| \dot{C}$ is precipitous. The argument for strong and presaturated ideals is left to the reader. 
Finally, let us verify that $\vdash_{P} \bar{I} \mid \dot{C}$ is normal. Let $\dot{A}$ be as above, and suppose $p \Vdash$ " $\dot{h}$ is regressive on $\dot{A}$ but $\dot{h}^{-1}(\{\alpha\}) \in \bar{I}$ for all $\alpha$ ". Now for each $\alpha \in \omega_{1}$ let $D_{\alpha}=\{q \leqslant p$ : $\left.\exists N(q, \alpha) \in I\left(q \Vdash \dot{h}^{-1}(\{\alpha\}) \subseteq N(q, \alpha)\right)\right\}$. By hypothesis, $D_{\alpha}$ is dense below $p$. For each $\alpha$ let $N_{\alpha}=\bigcup\left\{N(q, \beta): \beta<\alpha, q \in D_{\beta}, q \subseteq \alpha \times \alpha\right\}$. Then $N_{\alpha} \in I$ so by normality $N=\bigcup\left\{N_{\alpha}-(\alpha+1): \alpha \in \omega_{1}\right\} \in I$.

Let $E=\left\{\alpha \in \omega_{1}: \forall \beta<\alpha \forall q<p\right.$ if $q \subseteq \alpha \times \alpha$ then $\exists r \leqslant q(r \subseteq \alpha \times \alpha$ and $r \in$ $\left.\left.D_{\beta}\right)\right\}$. Then $E$ is closed unbounded so $E \in I^{*}$. Let $f(\alpha)=p \wedge\|\alpha \in \dot{A}\|$ as before, and let $A_{0}=\{\alpha: f(\alpha)$ and $\{(\alpha, \alpha)\}$ are compatible $\}$. We can find $\alpha \in\left(A_{0} \cap E\right)-N$. Fix $q \in P$ and $\beta$ such that $q \leqslant f(\alpha),\{(\alpha, \alpha)\}$ and $q$ ト $\dot{h}(\alpha)=\beta$. If $q^{\prime}=q \cap(\alpha \times \alpha)$ then since $\alpha \in E$ and $\beta<\alpha$ there is $r \leqslant q^{\prime}$ such that $r \subseteq \alpha \times \alpha$ and $r \in D_{\beta}$. Then $N(r, \beta) \subseteq N_{\alpha}$ so $\alpha \notin N(r, \beta)$. But $r$ and $g$ are compatible (since $q(\alpha)=\alpha$ and we may assume $\alpha$ is indecomposable) so $r \cup g \Vdash \alpha \in N(r, \beta)$, contradiction. Hence $\left|\vdash_{P} \bar{I}\right| \dot{C}$ is normal.

THEOREM 5.10. (a) If ZFC + "there is a presaturated ideal on $\omega_{1}$ " is consistent, then so is ZFC + "there is a presaturated ideal on $\omega_{1}$ but no $\omega_{2}$-saturated ideals on $\omega_{1}$ ".

(b) If $\mathrm{ZFC}+$ "there is a precipitous ideal on $\omega_{1}$ " is consistent, then so is ZFC + "there is a precipitous ideal on $\omega_{1}$ but no strong ideals (and hence no presaturated ideals) on $\omega_{1}$ ”.

Proof. For (a), let $I$ be presaturated and force with the ordering $P$ for adding a closed unbounded subset of $\omega_{1}$ with finite conditions. By Theorem 5.9, some restriction of $\bar{I}$ is presaturated, but by Theorem 3.5 there are no $\omega_{2}$-saturated ideals on $\omega_{1}$ in the generic extension.

(b) is included here only for completeness; it follows immediately from the remarks in the paragraph after Definition 5.6.

Theorem 5.10(a) yields a negative answer to the question asked following Corollary III.4.8 of [BTW].

In Theorem 5.9 we say that if $I$ is precipitous and normal and $P$ is the ordering for adding a closed unbounded subset of $\omega_{1}$ with finite conditions, then $\left|r_{P} \bar{I}\right| \dot{C}$ is precipitous. It is natural to ask whether $t_{P} \bar{I}$ is precipitous, as would be the case if $P$ had the countable chain condition. The following theorem shows that this is not true.

TheOREM 5.11. Let $P, G$ and $C$ be as in Theorem 5.9. If $I$ is a normal ideal on $\omega_{1}$, then $\vdash_{P}$ “ $\bar{I} \mid\left(\omega_{1}-\dot{C}\right)$ is nowhere precipitous".

Proof. Let $J=\bar{I} \mid\left(\omega_{1}-C\right)$ and note that $J$ is a proper countably complete ideal on $\omega_{1}$. It will suffice to show in $V[G]$ that

$\mid{ }_{B(J)}$ " there is no $\omega_{1}^{V[G]}$ th element in the generic ultrapower of $V[G]$ ".

For this, it will suffice to show that if $A \in J^{+}, f: A \rightarrow \omega_{1}$ and $f^{-1}(\{\alpha\}) \in J$ for every $\alpha<\omega_{1}$, then there exists $B \subseteq A$ and there exists $g: B \rightarrow \omega_{1}$ such that $B \in J^{+}$, $g^{-1}(\{\alpha\}) \in J$ for every $\alpha<\omega_{1}$ and $g(\alpha)<f(\alpha)$ for every $\alpha \in B$. Moreover, without loss of generality we may assume $A \cap C=0$ and $f(\alpha) \leqslant \max \{\beta<\alpha: \beta \in C\}$ for every $\alpha \in A$, since otherwise we could replace $f(\alpha)$ by $\min (f(\alpha), \max \{\beta<\alpha$, $\beta \in C\})$. Hence $f(\gamma)<\gamma$ for every $\gamma \in A$. 
Suppose $p_{0} \in P$ forces all the information about $A$ and $f$ in the preceding paragraph (with names $\dot{A}$ and $\dot{f}$ for $A$ and $f$ ).

Lemma 5.12. $\forall p \leqslant p_{0} \forall \alpha \exists \beta>\alpha\{\gamma: \exists q \leqslant p q \Vdash \gamma \in \dot{A}$ and $f(\gamma)=\beta\} \in I^{+}$.

Proof. Suppose not. Let $p$ and $\alpha$ be a counterexample, and for $\beta>\alpha$ let $X_{\beta}=\{\gamma$ : $\exists q \leqslant p q \Vdash \gamma \in \dot{A}$ and $\dot{f}(\gamma)=\beta\}$. Let $X=\cup\left\{X_{\beta}-(\beta+1): \beta>\alpha\right\}$. Since $I$ is normal, $X \in I$. But now $p_{0} \Vdash \forall \gamma \in \dot{A} \dot{f}(\gamma)<\gamma$, so we must have $p \Vdash \dot{f}: \dot{A}-X \rightarrow \alpha+1$ a contradiction since $p_{0} \Vdash \vdash^{\prime} \forall \beta \dot{f}^{-1}(\{\beta\}) \in J$ and $J$ is a countably complete proper ideal".

Now, working in $V$, let $E=\left\{\alpha<\omega_{1}: \forall p \leqslant p_{0} \forall \beta<\alpha\right.$ if $p \subseteq \alpha \times \alpha$ then $\exists \gamma \beta<\gamma$ $<\alpha$ and $X_{\gamma}=\{\delta: \exists q \leqslant p q \Vdash \delta \in \dot{A}$ and $\left.\dot{f}(\delta)=\gamma\} \in I^{+}\right\}$. Then $E$ is easily seen to be closed unbounded. If $\bar{E}$ is the set of limit points of $E$, then $\bar{E}$ is also closed unbounded.

Lemma 5.13. $p_{0} \Vdash\{\gamma \in \dot{A}: \dot{f}(\gamma) \notin E\} \in J^{+}$.

Proof. Let $p \leqslant p_{0}$ and $Z \in I$. Choose $\alpha \in \bar{E}$ so large that $p \subseteq \alpha \times \alpha$. Since $\bar{E}$ is the set of limit points of $E$, there is $\beta \in E-\bar{E}$ with $p \subseteq \beta \times \beta$. By definition of $E$ the set of $\gamma<\beta$ such that $X_{\gamma} \in I^{+}$is cofinal in $\beta$ and since $\beta$ is a successor point of $E$ there is such $\gamma \notin E$. Thus we can find $\delta \in X_{\gamma}-Z$ and $q \leqslant p$ such that $q \Vdash \dot{f}(\delta)=$ $\gamma \notin E$. This proves the lemma.

But now we are done, since in $V[G]$ we may let $B=\{\gamma \in A: f(\gamma) \notin E\}$ and define $g(\gamma)=\max \{\alpha \in E: \alpha<f(\gamma)\}$. It is easy to see that $B$ and $g$ are as desired, thus completing the proof of Theorem 5.11.

We conclude this section with a brief discussion of the Axiom A orderings. Recall that $(P, \leqslant)$ satisfies Axiom A iff there exist partial orderings $\leqslant_{n}, n \in \omega$ such that

(1) $p \leqslant q$ iff $p \leqslant 0$,

(2) $p \leqslant_{n+1} q$ implies $p \leqslant_{n} q$,

(3) if $A \subseteq P$ is pairwise incompatible, $p \in P$ and $n \in \omega$, then there exists $q \leqslant p$ such that $q \leqslant_{n} p$ and $\{r \in A: r, q$ are compatible $\}$ is countable,

(4) if $\left\langle p_{n}: n \in \omega\right\rangle$ is such that $p_{n+1} \leqslant_{n} p_{n}$ for all $n$, then there exists $p \in P$ such that $p \leqslant_{n} p_{n}$ for each $n \in \omega$.

If $P$ is countably closed or has the countable chain condition, then $P$ satisfies Axiom A. Other examples of Axiom A orderings are those due to Sacks, Silver, Mathias and Laver for adding real numbers. Further details are in $\left[\mathbf{B}_{\mathbf{2}}\right]$.

It will be convenient to have some ad hoc terminology.

Definition 5.14. If $I$ is a countably complete ideal on $\omega_{1}$, then we say $I$ has property $A$ iff

(5) $M$ has an $\omega_{1}^{\nu}$ th ordinal, and

(6) $(\exists f, G \in M) f: \omega \rightarrow \omega_{1}^{V}$ is a bijection and $G$ is $P_{0}$-generic over $V[f]$, where $P_{0}$ is the Cohen ordering for adding a subset of $\omega$ with finite conditions.

(Of course we really mean that (5) and (6) are forced by the ordering $B(I)$.)

An example of an ideal with property $A$ is the one constructed by Woodin in [W]. There $B(I)$ has a dense subset isomorphic to the standard collapse $P_{1}$ of $\omega_{1}$ onto $\omega$ with finite conditions. Since forcing with $P_{1}$ is the same as forcing with $P_{1} \times P_{0}$, and 
since for this ideal $V[U]$ and $M$ have the same reals, it is easy to see that the ideal has property $A$.

Theorem 5.15. If I has property $A, P$ satisfies Axiom $\mathrm{A}$, and $|P| \leqslant \omega_{1}$, then $P$ is I-regular.

Proof. It will suffice to prove the theorem under the assumption that $P \subseteq H C$, the collection of hereditarily countable sets.

LEMMA 5.16. If $(R, \leqslant)$ is an arbitrary partial ordering with $|R|=\omega_{1}, R \subseteq H C$, then $\exists \bar{G} \in M(\bar{G}$ is $R$-generic over $V)$.

Proof. Clearly $(R, \leqslant) \in M$. If $(\exists r \in R)\left(\forall q_{1}, q_{2} \leqslant r\right)\left(q_{1}\right.$ and $q_{2}$ are compatible), then $\bar{G}=\{q \in R: q$ is compatible with $r\}$ will work. Otherwise, there is a one-one mapping $h$ carrying $R$ into a dense subset of $P_{0}$ such that $h$ is constructed from the $f$ of (6) and $h$ is sufficiently canonical so that $h \in M \cap V[f]$. But now if $G \in M$ is as in (6) then $G$ gives rise to a set $\bar{G} \in M$ which is $R$-generic over $V$.

Now construct a partial ordering $R(P)$ as follows. Let $s \in R(P)$ iff $s \in$ $\cup\left\{{ }^{n} P: n \in \omega\right\}$ and $(\forall i+1 \in \operatorname{domain}(s))\left(s(i+1) \leqslant_{i} s(i)\right)$. Let $s \leqslant t$ iff $s \supseteq t$. Then Lemma 5.16 applies to $R(P)$ so there is a set $\bar{G} \in M$ which is $R(P)$-generic. Let $\left\langle p_{n}: n \in \omega\right\rangle=\cup \bar{G}$. This sequence satisfies the hypothesis of (4) in $j(P)$, so $\exists q \in j(P) \forall n q \leqslant_{n} p_{n}$.

LEMMA 5.17. $P$ is regular below $q$.

Proof. Let $D \in V$ be dense in $P$ and let $A \in V$ be a maximal incompatible subset of $D$. Let $D^{\prime}=\{s \in R(P): \exists i\{p \in A: s(i)$ is compatible with $p\}$ is countable $\}$. By (3), $D^{\prime}$ is dense in $R(P)$. Hence if $\left\langle p_{n}: n \in \omega\right\rangle=\cup \bar{G}$ as above, then for some $n$ and some countable $X \in V,\left\{p \in A: p\right.$ is compatible with $\left.p_{n}\right\} \subseteq X$. Now $j(A)$ is maximal incompatible in $M$, and $\left\{p \in j(A): p\right.$ is compatible with $\left.j\left(p_{n}\right)=p_{n}\right\} \subseteq j(X)=X$. Hence $(\exists p \in X) p(=j(p))$ is compatible with $q$, and $P$ is regular below $q$.

REMARKS. 1. Theorem 5.15 is only meaningful if $\mathrm{CH}$ holds in $V$ (as it does in Woodin's model) since all the interesting Axiom A orderings have cardinality $2^{\omega}$.

2 . We do not know of any ideal satisfying (5) which does not have property $A$. On the other hand there is a partial ordering $P$ which collapses $\omega_{1}$ (the Namba-style ordering of $\omega_{1}$-branching trees of height $\omega$ ), yet does not introduce any sets generic with respect to the Cohen ordering for adding a subset of $\omega_{1}$ with countable conditions. If there were an ideal $I$ such that $B(I)$ and $P$ had isomorphic dense subsets, then $I$ could not have property $A$.

6. Questions. In this final section we gather together several questions alluded to earlier in this paper. A reasonable starting point is with the single question that inspired much of our work here, but yet remains open.

Question 6.1. Is the $\omega_{2}$-saturation of a countably complete ideal on $\omega_{1}$ preserved under c.c.c. forcing?

Of course, the results of $\$ 2$ give several equivalent versions of this question. We explicitly state here only the following two. 
Question 6.2. Can $\omega_{1}$ carry a countably complete $\omega_{2}$-saturated ideal satisfying Chang's conjecture?

Question 6.3. Does $M A_{\omega_{2}}$ imply that there are no $\omega_{2}$-saturated ideals on $\omega_{1}$ ?

Theorem 2.2 suggests a general type of question which asks about the relationship between $V^{\omega_{1}} / U$ and $V[U]$ for the case where $U$ is $B(I)$-generic and $I$ is $\omega_{2}$-saturated. For example, the following does not seem to be obvious.

Question 6.4. Suppose that $I$ is $\omega_{2}$-saturated and that $S$ is a stationary set in (the transitive collapse) of $V^{\omega_{1}} / U$. Is $S$ stationary in $V[U]$ ?

The considerations of $\S 4$ leave open several questions; we mention the following three.

Question 6.5 . Is every $\omega_{2}$-preserving countably complete ideal on $\omega_{1}$ precipitous?

Question 6.6. Can one prove that an $\omega_{2}$-preserving countably complete ideal on $\omega_{1}$ must be a $P$-point?

Question 6.7. If $I$ is a presaturated ideal on $\omega_{1}$, can one find a normal presaturated ideal $J$ on $\omega_{1}$ so that $J \leqslant_{R K} I$ ?

$\S 5$ yields even more, the first of which is particularly tempting.

Question 6.8. Suppose that $P$ is a partial ordering so that forcing with $P$ preserves $\omega_{1}$. Does it follow that every countably complete ideal on $\omega_{1}$ in the ground model generates a proper countably complete ideal in the extension?

Note that for a negative answer we must have $2^{\omega_{1}}>\omega_{\omega}$, since condition $(*)$ at the beginning of $\S 5$ holds whenever $\alpha<\omega_{\omega}$ and $p \Vdash \dot{a} \subseteq \alpha$.

Recall that a countably complete ideal $I$ on $\omega_{1}$ is strong if $I$ is precipitous and $\vdash_{B(I)} j\left(\omega_{1}^{V}\right)=\omega_{2}^{V}$.

Question 6.9. Is every strong ideal presaturated?

Question 6.10. Does the existence of a strong ideal imply the existence of a normal strong ideal?

Question 6.11. Is the consistency of the existence of a strong ideal on $\omega_{1}$ equivalent to the consistency of the existence of an $\omega_{2}$-saturated ideal on $\omega_{1}$ ?

Question 6.12. Is the presaturation of a countably complete ideal on $\omega_{1}$ preserved under c.c.c. forcing?

\section{REFERENCES}

[BF] B. Balcar and F. Franek, Completion of factor Boolean algebras, handwritten manuscript.

$\left[\mathbf{B}_{1}\right]$ J. Baumgartner, Independence results in set theory, Notices Amer. Math. Soc. 25 (1978), A248-249.

$\left[\mathbf{B}_{\mathbf{2}}\right] \ldots$, Iterated forcing, Proc. 1978 Cambridge Summer School in Logic (to appear).

[BT] J. Baumgartner and A. Taylor, Saturation properties of ideals in generic extensions. I, Trans. Amer. Math. Soc. 270 (1982), 557-574.

[BTW] J. Baumgartner, A. Taylor and S. Wagon, Structural properties of ideals, Dissertationes Math. (to appear).

[J] T. Jech, Set theory, Academic Press, New York, 1978.

[JMMP] T. Jech, M. Magidor, W. Mitchell and K. Prikry, Precipitous ideals, J. Symbolic Logic 45 (1980), 1-8.

[JP] T. Jech and K. Prikry, Ideals over uncountable sets: application of almost disjoint functions and generic ultrapowers, Mem. Amer. Math. Soc., No. 214 (1979).

[K] Y. Kakuda, On a condition for Cohen extensions which preserve precipitous ideals (preprint).

$\left[\mathbf{K u}_{1}\right]$ K. Kunen, Some applications of iterated ultrapowers in set theory, Ann. Math. Logic 1 (1970), $179-227$. 
$\left[\mathbf{K u}_{2}\right] \ldots$ Saturated ideals, J. Symbolic Logic 43 (1978), 65-76.

[S] R. Solovay, Real valued measurable cardinals, Axiomatic Set Theory, Proc. Sympos. Pure Math., vol. 13, Amer. Math. Soc., Providence, R. I., 1971, pp. 397-428.

[T $\left.\mathbf{T}_{1}\right]$ A. Taylor, Regularity properties of ideals and ultrafilters, Ann. Math. Logic 16 (1979), 33-55.

$\left[\mathbf{T}_{\mathbf{2}}\right] \ldots$, On saturated sets of ideals and Ulam's problem, Fund. Math. 109 (1980), 37-53.

[VW] R. Van Wesep, The non-stationary ideal on $\omega_{1}$ can be $\omega_{2}$-saturated, handwritten manuscript.

[W] H. Woodin, An $\boldsymbol{\aleph}_{1}$-dense $\boldsymbol{\aleph}_{1}$-complete ideal on $\boldsymbol{\aleph}_{1}$, handwritten manuscript.

Department of Mathematics, Dartmouth College, Hanover, New Hampshire 03755

Department of Mathematics, Union College, Schenectady, New York 12308 